\title{
Stability results for the time-harmonic Maxwell equations with impedance boundary conditions
}

Article

Accepted Version

Hiptmair, R., Moiola, A. and Perugia, I. (2011) Stability results for the time-harmonic Maxwell equations with impedance boundary conditions. Mathematical models and methods in applied Sciences (M3AS), 21 (11). pp. 2263-2287. ISSN 02182025 doi: https://doi.org/10.1142/S021820251100574X Available at https://centaur.reading.ac.uk/28024/

It is advisable to refer to the publisher's version if you intend to cite from the work. See Guidance on citing.

To link to this article DOI: http://dx.doi.org/10.1142/S021820251100574X

Publisher: World Scientific

All outputs in CentAUR are protected by Intellectual Property Rights law, including copyright law. Copyright and IPR is retained by the creators or other copyright holders. Terms and conditions for use of this material are defined in the End User Agreement.

www.reading.ac.uk/centaur 
Central Archive at the University of Reading

Reading's research outputs online 
Mathematical Models and Methods in Applied Sciences

(C) World Scientific Publishing Company

\title{
STABILITY RESULTS FOR THE TIME-HARMONIC MAXWELL EQUATIONS WITH IMPEDANCE BOUNDARY CONDITIONS
}

\author{
RALF HIPTMAIR \\ Seminar for Applied Mathematics, ETH Zürich, Rämistrasse 101 \\ 8092 Zürich, Switzerland \\ ralf.hiptmair@sam.math.ethz.ch \\ ANDREA MOIOLA \\ Seminar for Applied Mathematics, ETH Zürich, Rämistrasse 101 \\ 8092 Zürich, Switzerland \\ andrea.moiola@sam.math.ethz.ch \\ ILARIA PERUGIA \\ Department of Mathematics, University of Pavia, Via Ferrata 1 \\ 27100 Pavia, Italy \\ ilaria.perugia@unipv.it \\ Received (Day Month Year) \\ Revised (Day Month Year) \\ Communicated by (xxxxxxxxxx)
}

\begin{abstract}
We consider the time-harmonic Maxwell equations with constant coefficients in a bounded, uniformly star-shaped polyhedron. We prove wavenumber-explicit norm bounds for weak solutions. This result is pivotal for convergence proofs in numerical analysis and may be a tool in the analysis of electromagnetic boundary integral operators.
\end{abstract}

Keywords: Time-harmonic Maxwell's equations; impedance boundary conditions; stability estimates; regularity.

AMS Subject Classification: 35B65, 35D30, 35Q61

\section{Introduction}

Stability estimates of variational solutions to PDE's with stability constants which are explicit in some of the characteristic parameters are important in the theoretical analysis, and then in the design, of discretization methods. Often, discretization parameters have to be chosen in relation to the physical ones, in order to design accurate, robust, and efficient numerical methods. This is the case for time-harmonic wave propagation problems, where the choice of the discretization parameters in relation to the wavenumber is crucial. There, fundamental model problems consider bounded domains with piecewise smooth boundary and first order absorbing 
boundary conditions (impedance boundary conditions, IBC).

For the Helmholtz problem with IBC, stability estimates in weighted $H^{1}$-norm with explicit dependence on the wavenumber were derived in Proposition 8.1.4 of [17] in the $2 \mathrm{D}$ case, then extended to the $3 \mathrm{D}$ case, with a similar argument, in [7] and [11]; in the latter reference, the case of mixed boundary conditions was also considered. In these results, in order to use Rellich identities, the problem domain is assumed to be star-shaped with respect to a ball; a key ingredient in the proof is the fact that the weak solution is smoother than merely $H^{1}$, which holds true for polygonal/polyhedral domains.

For the time-harmonic Maxwell equations with IBC, stability estimates were derived with a Fredholm-type argument in [18, Theorem 4.17]. Unfortunately, this analysis does not allow to establish how the stability constant depends on the wavenumber. The main obstacle to extending the argument of [17] to the Maxwell case consists in the poor regularity of the analytical solutions, even in the case of constant material coefficients. In fact, while for Dirichlet boundary conditions, the solution always has $H^{1}$-regularity in convex domains, for IBC, $H^{1}$-regularity is guaranteed only for smooth domains (see [8]).

In this paper, we consider the time-harmonic Maxwell equations with constant coefficients in bounded, uniformly star-shaped domains. In Section 3 stability estimates in a weighted $H$ (curl)-norm are derived. For smooth domains, relying upon the regularity results established in [8], we extend the argument of [17] and prove stability with constants independent of the wavenumber (see Theorem 3.1). Then, with a technique similar to that of [10, Thm. 3.2.1.3], we extend this result to polyhedral domains (see Theorem 3.2).

For the analysis of numerical approximations of Maxwell solutions, which relies on duality arguments, it is also interesting to derive elliptic regularity results. For this reason, in Section 4 (see Theorem 4.4), we prove that, provided that the boundary data are in $H^{s^{\prime}}, 0<s^{\prime}<1 / 2$, the solutions reach a regularity $H^{1 / 2+s}$, for some $0<s \leq s^{\prime}<1 / 2$, in polyhedral domains. In a convex polyhedron, the regularity is always optimal: $s=s^{\prime}<1 / 2$. The constant in the stability estimates in stronger norms ( $H^{1}$ for smooth domains, $H^{1 / 2+s}$ for polyhedral domains) linearly depends on the wavenumber.

Our main reason of interest in these stability and regularity results was their application in the error analysis of Trefftz-discontinuous Galerkin approximations of the time-harmonic Maxwell equations. In fact, in [13], we are extending to the Maxwell case the theory developed in [14] for the Helmholtz problem, where uniform stability with respect to the wavenumber, together with elliptic regularity, played an essential role. Another potential application is the extension to electromagnetic waves of the norm and stability bounds of boundary integral operators for acoustic scattering derived in [6] and [23].

We conclude this introduction by setting some notation used throughout this paper. If $D$ is a domain in $\mathbb{R}^{2}$ or $\mathbb{R}^{3}$, we denote by $H^{k}(D)^{d}, d=1,2,3$, the Sobolev 
space with integer or fractional regularity index $k$ and values in $\mathbb{C}^{d}$, and by $\|\cdot\|_{k, \Omega}$ the corresponding Sobolev norm; we denote by $H_{0}^{1}(D)$ the closure in $H^{1}(D)$ of $C_{0}^{\infty}(D)$ and set $L^{2}(D)=H^{0}(D)$.

For $D \subset \mathbb{R}^{3}$, we introduce the following Hilbert spaces, see also [9, Ch. 1],

$$
\begin{aligned}
& H(\operatorname{curl} ; D)=\left\{\boldsymbol{v} \in L^{2}(D)^{3}: \nabla \times \boldsymbol{v} \in L^{2}(D)^{3}\right\}, \\
& H(\operatorname{div} ; D)=\left\{\boldsymbol{v} \in L^{2}(D)^{3}: \nabla \cdot \boldsymbol{v} \in L^{2}(D)\right\} \\
& H\left(\operatorname{div}^{0} ; D\right)=\left\{\boldsymbol{v} \in L^{2}(D)^{3}: \nabla \cdot \boldsymbol{v}=0 \text { in } D\right\} \\
& L_{T}^{2}(\partial D):=\left\{\boldsymbol{v} \in L^{2}(\partial D)^{3}: \boldsymbol{v} \cdot \boldsymbol{n}=0\right\}
\end{aligned}
$$

endowed with the corresponding graph norms.

If $D$ is a Lipschitz domain in $\mathbb{R}^{3}$ and $\boldsymbol{n}$ is the exterior unit normal vector field to $\partial D$, the following integration by parts formula holds true for functions in $H(\operatorname{curl} ; D)$ :

$$
\int_{D} \nabla \times \boldsymbol{F} \cdot \overline{\boldsymbol{G}} d V=\int_{D} \boldsymbol{F} \cdot \overline{\nabla \times \boldsymbol{G}} d V+\int_{\partial D} \boldsymbol{n} \times \boldsymbol{F} \cdot \overline{\boldsymbol{G}} d S
$$

provided that the second integral on the right-hand side is intended as a duality product between the appropriate trace spaces (see [3]). If $\boldsymbol{D}$ is a vector-valued function defined in $D$, we denote its normal and tangential components on $\partial D$ by $\boldsymbol{D}_{N}:=(\boldsymbol{D} \cdot \boldsymbol{n}) \boldsymbol{n}$ and $\boldsymbol{D}_{T}:=(\boldsymbol{n} \times \boldsymbol{D}) \times \boldsymbol{n}$, respectively.

Finally, we write $B_{r}\left(\boldsymbol{x}_{0}\right)$ for the (open) ball of radius $r$ centered at $\boldsymbol{x}_{0}$ and by $\mathbb{S}^{2}=\left\{\boldsymbol{x} \in \mathbb{R}^{3}:|\boldsymbol{x}|=1\right\}$ the unit sphere.

\section{The Maxwell boundary value problem}

Let $\Omega \subset \mathbb{R}^{3}$ be an open bounded domain, which is either has a $C^{2}$ boundary or is a polyhedron. We assume that

there exist a point $\boldsymbol{x}_{0} \in \Omega$ and a real number $\gamma>0$ for which $\Omega$ is star-shaped with respect to all points in $B_{\gamma}\left(\boldsymbol{x}_{0}\right)$.

For each point $\boldsymbol{x} \in \partial \Omega$, the open cone with vertex $\boldsymbol{x}$, height $\left|\boldsymbol{x}-\boldsymbol{x}_{0}\right|$ and opening angle $\theta=\arctan \left(\gamma /\left|\boldsymbol{x}-\boldsymbol{x}_{0}\right|\right)>\arctan (\gamma / \operatorname{diam}(\Omega))$ is contained in $\Omega$. This means that the domain satisfies the uniform cone condition; therefore, by [10, Thm. 1.2.2.2], $\Omega$ is Lipschitz.

We consider the following frequency-domain formulation of the Maxwell equations in terms of electric field $\boldsymbol{E}$ and magnetic field $\boldsymbol{H}$ with impedance boundary conditions in the domain $\Omega$ :

$$
\begin{cases}-i \omega \epsilon \boldsymbol{E}-\nabla \times \boldsymbol{H}=-\boldsymbol{J} / i \omega & \text { in } \Omega, \\ -i \omega \mu \boldsymbol{H}+\nabla \times \boldsymbol{E}=\mathbf{0} & \text { in } \Omega, \\ \boldsymbol{H} \times \boldsymbol{n}-\lambda(\boldsymbol{n} \times \boldsymbol{E}) \times \boldsymbol{n}=\boldsymbol{g} / i \omega & \text { on } \partial \Omega\end{cases}
$$

where $\omega>0$ is a fixed wave number, $\boldsymbol{J} \in H\left(\operatorname{div}^{0} ; \Omega\right)$ is related to a given current density, and $\boldsymbol{g} \in L_{T}^{2}(\partial \Omega)$. The material coefficients 


$$
\epsilon, \mu, \lambda \in \mathbb{R} \text { are assumed to be constant }
$$

with $\epsilon, \mu>0$ and $\lambda \neq 0$.

By expressing $\boldsymbol{H}$ in terms of $\boldsymbol{E}$ using the second equation of (2.1) and replacing into the first equation and into the boundary condition, we obtain

$$
\begin{cases}\nabla \times\left(\mu^{-1} \nabla \times \boldsymbol{E}\right)-\omega^{2} \epsilon \boldsymbol{E}=\boldsymbol{J} & \text { in } \Omega, \\ \left(\mu^{-1} \nabla \times \boldsymbol{E}\right) \times \boldsymbol{n}-i \omega \lambda(\boldsymbol{n} \times \boldsymbol{E}) \times \boldsymbol{n}=\boldsymbol{g} & \text { on } \partial \Omega .\end{cases}
$$

Introducing the "energy space" (equipped with graph norm)

$$
H_{\mathrm{imp}}(\operatorname{curl} ; \Omega)=\left\{\boldsymbol{v} \in H(\operatorname{curl} ; \Omega): \boldsymbol{v}_{T} \in L_{T}^{2}(\partial \Omega)\right\},
$$

the variational formulation of the Maxwell problem (2.2) reads as follows: find $\boldsymbol{E} \in H_{\mathrm{imp}}(\operatorname{curl} ; \Omega)$ such that, for all $\boldsymbol{\xi} \in H_{\mathrm{imp}}(\operatorname{curl} ; \Omega)$, it holds

$$
\mathcal{A}(\boldsymbol{E}, \boldsymbol{\xi})=\int_{\Omega} \boldsymbol{J} \cdot \overline{\boldsymbol{\xi}} \mathrm{d} V+\int_{\partial \Omega} \boldsymbol{g} \cdot \overline{\boldsymbol{\xi}}_{T} \mathrm{~d} S
$$

where

$$
\mathcal{A}(\boldsymbol{E}, \boldsymbol{\xi}):=\int_{\Omega}\left[\left(\mu^{-1} \nabla \times \boldsymbol{E}\right) \cdot(\overline{\nabla \times \boldsymbol{\xi}})-\omega^{2}(\epsilon \boldsymbol{E}) \cdot \overline{\boldsymbol{\xi}}\right] \mathrm{d} V-i \omega \int_{\partial \Omega} \lambda \boldsymbol{E}_{T} \cdot \overline{\boldsymbol{\xi}}_{T} \mathrm{~d} S .
$$

Well-posedness of problem (2.3) in $H_{\mathrm{imp}}(\operatorname{curl} ; \Omega)$ is proved in [18, Thm. 4.17] that we report here.

Theorem 2.1. Under the assumptions made on $\Omega, \boldsymbol{J}, \boldsymbol{g}$ and on the material coefficients, there exists a unique $\boldsymbol{E} \in H_{\text {imp }}(\operatorname{curl} ; \Omega)$ with $\nabla \cdot(\epsilon \boldsymbol{E})=0$ solution to (2.3).

\section{Stability estimates}

In this section, we prove stability estimates in energy-norm for problem (2.3), with stability constants independent of the wave number $\omega$.

We use the argument developed in [17, Sect. 8.1] (see also [7] and [11]) for the Helmholtz problem. In order to do that, we have to choose a particular test functions, the admissibility of which requires some smoothness of the Maxwell solution. Therefore, we will proceed in two steps: in Section 3.1 following [17, Proof of Prop. 8.1.4] and [11], we prove stability estimates for problem (2.3) for $C^{2}$-domains (see Theorem 3.1). Then, in Section 3.2 we extend this result to non-smooth domains (see Theorem [3.2). Before doing that, we establish the following geometric equivalence.

Lemma 3.1. Let $\Omega \subset \mathbb{R}^{3}$ be a bounded, either $C^{2}$ or polyhedral domain. Then $\Omega$ is star-shaped with respect to $B_{\gamma}\left(\boldsymbol{x}_{0}\right)$ if and only if, for all $\boldsymbol{x} \in \partial \Omega$ for which $\boldsymbol{n}(\boldsymbol{x})$ is defined, $\left(\boldsymbol{x}-\boldsymbol{x}_{0}\right) \cdot \boldsymbol{n}(\boldsymbol{x}) \geq \gamma$.

Proof. Set $\Gamma:=\{\boldsymbol{x} \in \partial \Omega: \boldsymbol{n}(\boldsymbol{x})$ is defined $\}$; our assumptions on $\Omega$ imply that $\partial \Omega \backslash \Gamma$ has zero $2-$ measure. 
If $\Omega$ is star-shaped with respect to $B_{\gamma}\left(\boldsymbol{x}_{0}\right)$ then, for all $\boldsymbol{x} \in \Gamma$, the tangent plane in $\boldsymbol{x}$ to $\partial \Omega$ does not intersects the (open) tangential cone to $\partial B_{\gamma}\left(\boldsymbol{x}_{0}\right)$ with vertex $\boldsymbol{x}$. Since $\left(\boldsymbol{x}-\boldsymbol{x}_{0}\right) \cdot \boldsymbol{n}(\boldsymbol{x})$ is equal to the signed distance of $\boldsymbol{x}_{0}$ from the tangent plane in $\boldsymbol{x}$ to $\partial \Omega$, then $\left(\boldsymbol{x}-\boldsymbol{x}_{0}\right) \cdot \boldsymbol{n}(\boldsymbol{x}) \geq \gamma$.

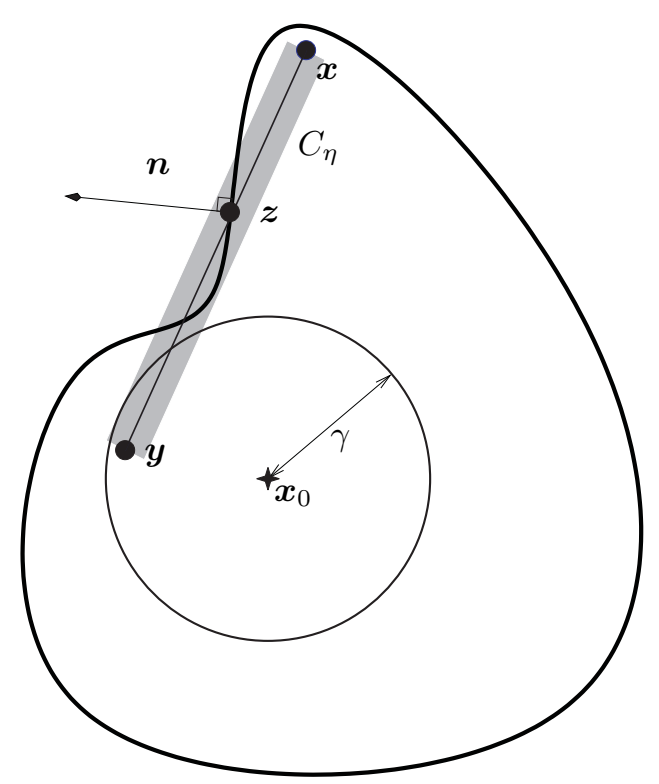

Fig. 1. Geometric considerations in the proof of Lemma 3.1

We prove the converse by contradiction; see Fig. 1 Assume that there exist $\boldsymbol{x} \in \Omega$ and $\boldsymbol{y} \in B_{\gamma}\left(\boldsymbol{x}_{0}\right)$ such that the segment $(\boldsymbol{x}, \boldsymbol{y})$ is not contained in $\Omega$. Then, there exists $\boldsymbol{z} \in(\boldsymbol{x}, \boldsymbol{y}) \cap \partial \Omega$ such that the open segment $(\boldsymbol{x}, \boldsymbol{z})$ is contained in $\Omega$. (i) If $\boldsymbol{z} \in \Gamma$, then $\left(\boldsymbol{z}-\boldsymbol{x}_{0}\right) \cdot \boldsymbol{n}(\boldsymbol{z})=(\boldsymbol{z}-\boldsymbol{y}) \cdot \boldsymbol{n}(\boldsymbol{z})+\left(\boldsymbol{y}-\boldsymbol{x}_{0}\right) \cdot \boldsymbol{n}(\boldsymbol{z})$; since $(\boldsymbol{z}-\boldsymbol{y}) \cdot \boldsymbol{n}(\boldsymbol{z}) \leq 0$ and $\left(\boldsymbol{y}-\boldsymbol{x}_{0}\right) \cdot \boldsymbol{n}(\boldsymbol{z})<\gamma \cdot 1$, then $\left(\boldsymbol{z}-\boldsymbol{x}_{0}\right) \cdot \boldsymbol{n}(\boldsymbol{z})<\gamma$, which contradicts the assumption. (ii) If $z \notin \Gamma$, there exists $\eta>0$ such that the (open, infinite) cylinder $C_{\eta}$ with axis through $\boldsymbol{x}$ and $\boldsymbol{y}$, and radius $\eta$ is such that its orthogonal sections $S_{\mathbf{x}}$ and $S_{\mathbf{y}}$ through $\boldsymbol{x}$ and $\boldsymbol{y}$, respectively, are contained in $\Omega$ and $B_{\gamma}\left(\boldsymbol{x}_{0}\right)$, respectively. Since $C_{\eta} \cap \Gamma$ is an open dense subset of $C_{\eta} \cap \partial \Omega$, let $\boldsymbol{z}^{\prime}$ be one of its points such that, defined $\boldsymbol{x}^{\prime}$ and $\boldsymbol{y}^{\prime}$ as the orthogonal projections of $\boldsymbol{z}^{\prime}$ onto $S_{\mathbf{x}}$ and $S_{\mathbf{y}}$, respectively, the points $\boldsymbol{x}^{\prime}, \boldsymbol{y}^{\prime}$ and $\boldsymbol{z}^{\prime}$ are in the same situation as the points $\boldsymbol{x}, \boldsymbol{y}$ and $\boldsymbol{z}$ in case (i). Then we conclude that $\left(\boldsymbol{z}^{\prime}-\boldsymbol{x}_{0}\right) \cdot \boldsymbol{n}\left(\boldsymbol{z}^{\prime}\right)<\gamma$, which contradicts the assumptiol

The assertion of Lemma 3.1 amounts to the identity

$$
\begin{aligned}
& \sup \left\{\gamma \in \mathbb{R}: \Omega \text { is star-shaped with respect to } B_{\gamma}\left(\boldsymbol{x}_{0}\right)\right\} \\
& =\inf \left\{\left(\boldsymbol{x}-\boldsymbol{x}_{0}\right) \cdot \boldsymbol{n}(\boldsymbol{x}): \boldsymbol{x} \in \partial \Omega \text { and } \boldsymbol{n}(\boldsymbol{x}) \text { is defined }\right\} .
\end{aligned}
$$




\subsection{Stability for smooth domains}

In this section, we consider the case of $C^{2}$-domains. This ensures that all the Sobolev spaces $H^{s}(\partial \Omega),-2<s<2$, and their tangential vectorial counterparts $H_{T}^{s}(\partial \Omega):=$ $\left\{\boldsymbol{\varphi} \in H^{s}(\partial \Omega)^{3}: \boldsymbol{\varphi} \cdot \boldsymbol{n}=0\right\}$ are well defined (see [1, p. 825]).

In order to prove stability estimates for problem (2.3), we need the following regularity result proved in $[8$, Sect. 4.5.d]. We report here the proof for the sake of completeness.

Lemma 3.2. Let $\Omega \subset \mathbb{R}^{3}$ be a bounded $C^{2}$-domain. In addition to the assumptions made on $\boldsymbol{J}, \boldsymbol{g}$ and on the material coefficients in Section 2 we assume $\boldsymbol{g} \in H_{T}^{1 / 2}(\partial \Omega)$. Then, the solution $\boldsymbol{E}$ to problem (2.3) belongs to $H^{1}(\operatorname{curl} ; \Omega):=$ $\left\{\boldsymbol{v} \in H^{1}(\Omega)^{3}: \nabla \times \boldsymbol{v} \in H^{1}(\Omega)^{3}\right\}$.

Proof. Decompose $\boldsymbol{E}$ as

$$
\boldsymbol{E}=\boldsymbol{\Phi}^{0}+\nabla \psi
$$

where $\boldsymbol{\Phi}^{0} \in H^{1}(\Omega) \cap H\left(\operatorname{div}^{0} ; \Omega\right)$ and $\psi \in H^{1}(\Omega)$ (see [12, Lemma 2.4]); clearly, $\Delta \psi=0$ in $\Omega$. By using this decomposition, we can write the boundary condition in problem (2.3) by

$$
\left(\mu^{-1} \nabla \times \boldsymbol{E}\right) \times \boldsymbol{n}-i \omega \lambda \boldsymbol{\Phi}_{T}^{0}-i \omega \lambda \nabla_{T} \psi=\boldsymbol{g} \quad \text { on } \partial \Omega,
$$

where $\nabla_{T} \psi$ is the tangential gradient of $\psi$, i.e., $\nabla_{T} \psi:=(\boldsymbol{n} \times \nabla \psi) \times \boldsymbol{n}$.

Using the results of [3] (see also [18, eq. (3.52)]), the tangential divergence $\operatorname{div}_{T}$ of $\left(\mu^{-1} \nabla \times \boldsymbol{E}\right) \times \boldsymbol{n}$ is well-defined, belongs to $H^{-1 / 2}(\partial \Omega)$. Moreover, $\boldsymbol{\Phi}_{T}^{0}, \boldsymbol{g} \in$ $H^{1 / 2}(\partial \Omega)^{3}$, and thus $\operatorname{div}_{T}\left(\lambda \boldsymbol{\Phi}_{T}^{0}+\boldsymbol{g}\right) \in H^{-1 / 2}(\partial \Omega)$. It follows that $\operatorname{div}_{T} \lambda \nabla_{T} \psi \in$ $H^{-1 / 2}(\partial \Omega)$ and, by an elliptic lifting theorem for the Laplace-Beltrami operator on smooth surfaces, we find $\psi \in H^{3 / 2}(\partial \Omega)$; this, together with $\Delta \psi=0$ in $\Omega$, gives $\psi \in H^{2}(\Omega)$, due to the smoothness of $\partial \Omega$, which implies $\boldsymbol{E} \in H^{1}(\Omega)^{3}$.

Similarly, we prove the smoothness of $\nabla \times \boldsymbol{E}$ : decompose $\nabla \times \boldsymbol{E}$ as

$$
\nabla \times \boldsymbol{E}=\boldsymbol{\Psi}^{0}+\nabla \phi
$$

where $\Psi^{0} \in H^{1}(\Omega)^{3} \cap H\left(\operatorname{div}^{0} ; \Omega\right)$, and $\phi \in H^{1}(\Omega)$; again, $\Delta \phi=0$ in $\Omega$. The boundary condition in problem (2.3) can be written as

$$
\mu^{-1} \Psi^{0} \times \boldsymbol{n}+\mu^{-1} \nabla \phi \times \boldsymbol{n}-i \omega \lambda \boldsymbol{E}_{T}=\boldsymbol{g} \quad \text { on } \partial \Omega .
$$

The tangential curl $\operatorname{curl}_{T} \boldsymbol{E}_{T}$ is well-defined and belongs to $H^{-1 / 2}(\partial \Omega)$. Moreover, $\boldsymbol{\Psi}^{0} \times \boldsymbol{n}, \boldsymbol{g} \in H_{T}^{1 / 2}(\partial \Omega)$. Thus, $\operatorname{curl}_{T}\left(\mu^{-1} \boldsymbol{\Psi}^{0} \times \boldsymbol{n}-\boldsymbol{g}\right) \in H^{-1 / 2}(\partial \Omega)$. Thus, since

$$
\operatorname{curl}_{T}\left(\mu^{-1} \nabla \phi \times \boldsymbol{n}\right)=-\operatorname{div}_{T}\left(\boldsymbol{n} \times\left(\mu^{-1} \nabla \phi \times \boldsymbol{n}\right)\right)=-\operatorname{div}_{T} \mu^{-1} \nabla_{T} \phi
$$

(see [18, Formula (3.15), p. 49]), we have that $\operatorname{div}_{T} \mu^{-1} \nabla_{T} \phi \in H^{-1 / 2}(\partial \Omega)$. Again, the regularity results for the Laplace-Beltrami operator confirm $\phi \in H^{3 / 2}(\partial \Omega)$, which, together with $\Delta \phi=0$, gives $\phi \in H^{2}(\Omega)$, and thus $\nabla \times \boldsymbol{E} \in H^{1}(\Omega)^{3}$.

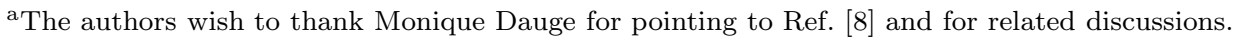


We are now ready to prove our stability result for smooth domains.

Theorem 3.1. Let $\Omega \subset \mathbb{R}^{3}$ be a bounded $C^{2}$-domain which is star-shaped with respect to $B_{\gamma}\left(\boldsymbol{x}_{0}\right)$, and let $\boldsymbol{J}, \boldsymbol{g}$ and the material coefficients satisfy the assumptions made in Section Q Then, there exist two positive constants $C_{1}, C_{2}$ independent of $\omega$, but depending on $d:=\operatorname{diam}(\Omega), \gamma, \lambda, \epsilon$ and $\mu$, such that, if $\boldsymbol{E}$ is the solution to (2.3),

$$
\left\|\mu^{-1 / 2} \nabla \times \boldsymbol{E}\right\|_{0, \Omega}+\omega\left\|\epsilon^{1 / 2} \boldsymbol{E}\right\|_{0, \Omega} \leq C_{1}\|\boldsymbol{J}\|_{0, \Omega}+C_{2}\|\boldsymbol{g}\|_{0, \partial \Omega} .
$$

Moreover, there exist two positive constants $C_{3}$ and $C_{4}$ independent of $\omega$, but depending on $d, \gamma, \lambda, \epsilon$ and $\mu$, such that

$$
\omega\left\|\lambda^{1 / 2} \boldsymbol{E}_{T}\right\|_{0, \partial \Omega} \leq C_{3}\|\boldsymbol{J}\|_{0, \Omega}+C_{4}\|\boldsymbol{g}\|_{0, \partial \Omega} .
$$

Proof. It is enough to prove the result in the case $\boldsymbol{g} \in H_{T}^{1 / 2}(\partial \Omega)$, then the general case $\boldsymbol{g} \in L_{T}^{2}(\partial \Omega)^{3}$ will follow by a density argument.

We assume, with no loss of generality, that $\boldsymbol{x}_{0}=\mathbf{0}$. Taking the imaginary part of $\mathcal{A}(\boldsymbol{E}, \boldsymbol{E})$ and using the Young inequality give

$$
\omega\left\|\lambda^{1 / 2} \boldsymbol{E}_{T}\right\|_{0, \partial \Omega}^{2} \leq\left|\int_{\Omega} \boldsymbol{J} \cdot \overline{\boldsymbol{E}} \mathrm{d} V\right|+\frac{\omega^{-1}}{2}\left\|\lambda^{-1 / 2} \boldsymbol{g}\right\|_{0, \partial \Omega}^{2}+\frac{\omega}{2}\left\|\lambda^{1 / 2} \boldsymbol{E}_{T}\right\|_{0, \partial \Omega}^{2},
$$

from which

$$
\omega^{2}\left\|\lambda^{1 / 2} \boldsymbol{E}_{T}\right\|_{0, \partial \Omega}^{2} \leq 2 \omega\left|\int_{\Omega} \boldsymbol{J} \cdot \overline{\boldsymbol{E}} \mathrm{d} V\right|+\left\|\lambda^{-1 / 2} \boldsymbol{g}\right\|_{0, \partial \Omega}^{2} .
$$

We proceed along the lines of the proof of [17, Prop. 8.1.4], and set $\boldsymbol{\xi}=(\nabla \times$ $\boldsymbol{E}) \times \boldsymbol{x}$, which is an admissible test function, since, thanks to Lemma $3.2 \boldsymbol{E} \in$ $H^{1}(\operatorname{curl} ; \Omega)$.

In order to compute $\operatorname{Re}[\mathcal{A}(\boldsymbol{E}, \boldsymbol{\xi})]$, observe that the identity

$$
\nabla \times(\boldsymbol{a} \times \boldsymbol{b})=\boldsymbol{a}(\nabla \cdot \boldsymbol{b})-\boldsymbol{b}(\nabla \cdot \boldsymbol{a})+(\boldsymbol{b} \cdot \nabla) \boldsymbol{a}-(\boldsymbol{a} \cdot \nabla) \boldsymbol{b},
$$

together with $\nabla \cdot \boldsymbol{x}=3$, gives

$$
\nabla \times \boldsymbol{\xi}=3 \nabla \times \boldsymbol{E}+(\boldsymbol{x} \cdot \nabla) \nabla \times \boldsymbol{E}-(\nabla \times \boldsymbol{E} \cdot \nabla) \boldsymbol{x} ;
$$

this, along with the identities

$$
\begin{aligned}
2 \operatorname{Re}[\boldsymbol{w} \cdot(\boldsymbol{x} \cdot \nabla) \overline{\boldsymbol{w}}] & =\boldsymbol{x} \cdot \nabla\left(|\boldsymbol{w}|^{2}\right), \\
(\boldsymbol{w} \cdot \nabla) \boldsymbol{x} & =\boldsymbol{w}
\end{aligned}
$$

gives

$$
\begin{aligned}
\operatorname{Re}[\mathcal{A}(\boldsymbol{E}, \boldsymbol{\xi})]= & 2\left\|\mu^{-1 / 2} \nabla \times \boldsymbol{E}\right\|_{0, \Omega}^{2}+\frac{1}{2} \int_{\Omega} \boldsymbol{x} \cdot \nabla\left(\left|\mu^{-1 / 2} \nabla \times \boldsymbol{E}\right|^{2}\right) \mathrm{d} V \\
& -\operatorname{Re}\left[\omega^{2} \int_{\Omega}(\epsilon \boldsymbol{E}) \cdot((\nabla \times \overline{\boldsymbol{E}}) \times \boldsymbol{x}) \mathrm{d} V\right] \\
& -\operatorname{Re}\left[i \omega \int_{\partial \Omega} \lambda \boldsymbol{E}_{T} \cdot((\nabla \times \overline{\boldsymbol{E}}) \times \boldsymbol{x})_{T} \mathrm{~d} S\right]
\end{aligned}
$$


Integrating by parts and recalling that $\nabla \cdot \boldsymbol{x}=3$, we get

$$
\begin{aligned}
\frac{1}{2} \int_{\Omega} \boldsymbol{x} \cdot \nabla\left(\left|\mu^{-1 / 2} \nabla \times \boldsymbol{E}\right|^{2}\right) \mathrm{d} V= & -\frac{3}{2}\left\|\mu^{-1 / 2} \nabla \times \boldsymbol{E}\right\|_{0, \Omega}^{2} \\
& +\frac{1}{2} \int_{\partial \Omega}(\boldsymbol{x} \cdot \boldsymbol{n})\left|\mu^{-1 / 2} \nabla \times \boldsymbol{E}\right|^{2} \mathrm{~d} V .
\end{aligned}
$$

Using the identity

$$
\nabla(\boldsymbol{a} \cdot \boldsymbol{b})=(\boldsymbol{a} \cdot \nabla) \boldsymbol{b}+(\boldsymbol{b} \cdot \nabla) \boldsymbol{a}+\boldsymbol{a} \times(\nabla \times \boldsymbol{b})+\boldsymbol{b} \times(\nabla \times \boldsymbol{a}),
$$

and taking into account that $\nabla \times \boldsymbol{x}=\mathbf{0}$, we obtain

$$
\begin{aligned}
& \operatorname{Re}\left[\omega^{2} \int_{\Omega}(\epsilon \boldsymbol{E}) \cdot((\nabla \times \overline{\boldsymbol{E}}) \times \boldsymbol{x}) \mathrm{d} V\right] \\
& =\operatorname{Re}\left[\omega^{2} \int_{\Omega}[(\epsilon \boldsymbol{E}) \cdot(\overline{\boldsymbol{E}} \cdot \nabla) \boldsymbol{x}+(\epsilon \boldsymbol{E}) \cdot(\boldsymbol{x} \cdot \nabla) \overline{\boldsymbol{E}}-(\epsilon \boldsymbol{E}) \cdot \nabla(\overline{\boldsymbol{E}} \cdot \boldsymbol{x})] \mathrm{d} V\right] \\
& =\omega^{2}\left\|\epsilon^{1 / 2} \boldsymbol{E}\right\|_{0, \Omega}^{2}+\frac{\omega^{2}}{2} \int_{\Omega} \boldsymbol{x} \cdot \nabla\left(\left|\epsilon^{1 / 2} \boldsymbol{E}\right|^{2}\right) \mathrm{d} V-\operatorname{Re}\left[\omega^{2} \int_{\Omega}(\epsilon \boldsymbol{E}) \cdot \nabla(\overline{\boldsymbol{E}} \cdot \boldsymbol{x})\right] \\
& =-\frac{\omega^{2}}{2}\left\|\epsilon^{1 / 2} \boldsymbol{E}\right\|_{0, \Omega}^{2}+\frac{\omega^{2}}{2} \int_{\partial \Omega}(\boldsymbol{x} \cdot \boldsymbol{n})\left|\epsilon^{1 / 2} \boldsymbol{E}\right|^{2} \mathrm{~d} S-\operatorname{Re}\left[\omega^{2} \int_{\partial \Omega}(\epsilon \boldsymbol{E}) \cdot \boldsymbol{n}(\overline{\boldsymbol{E}} \cdot \boldsymbol{x}) \mathrm{d} S\right],
\end{aligned}
$$

where the second identity is a consequence of the two formulas in (3.4), and the third one has been obtained integrating by parts and taking into account that $\nabla \cdot \boldsymbol{x}=3$ and $\nabla \cdot(\epsilon \boldsymbol{E})=0$.

Then (3.5) becomes

$$
\operatorname{Re}[\mathcal{A}(\boldsymbol{E}, \boldsymbol{\xi})]=\frac{1}{2}\left\|\mu^{-1 / 2} \nabla \times \boldsymbol{E}\right\|_{0, \Omega}^{2}+\frac{\omega^{2}}{2}\left\|\epsilon^{1 / 2} \boldsymbol{E}\right\|_{0, \Omega}^{2}+T 1+T 2,
$$

where

$$
\begin{aligned}
& T 1:=-\frac{\omega^{2}}{2} \int_{\partial \Omega}(\boldsymbol{x} \cdot \boldsymbol{n})\left|\epsilon^{1 / 2} \boldsymbol{E}\right|^{2} \mathrm{~d} S+\operatorname{Re}\left[\omega^{2} \int_{\partial \Omega}(\epsilon \boldsymbol{E}) \cdot \boldsymbol{n}(\overline{\boldsymbol{E}} \cdot \boldsymbol{x}) \mathrm{d} S\right], \\
& T 2:=\frac{1}{2} \int_{\partial \Omega}(\boldsymbol{x} \cdot \boldsymbol{n})\left|\mu^{-1 / 2} \nabla \times \boldsymbol{E}\right|^{2} \mathrm{~d} S-\operatorname{Re}\left[i \omega \int_{\partial \Omega} \lambda \boldsymbol{E}_{T} \cdot((\nabla \times \overline{\boldsymbol{E}}) \times \boldsymbol{x})_{T} \mathrm{~d} S\right] .
\end{aligned}
$$

The term $T 1$ can be estimated by using the splitting of vector-valued functions on $\partial \Omega$ into their normal and tangential components and the Young inequality with weight $\sqrt{\boldsymbol{x} \cdot \boldsymbol{n}}$ :

$$
\begin{aligned}
T 1= & -\frac{\omega^{2}}{2} \int_{\partial \Omega}(\boldsymbol{x} \cdot \boldsymbol{n})\left|\epsilon^{1 / 2} \boldsymbol{E}_{T}\right|^{2} \mathrm{~d} S+\frac{\omega^{2}}{2} \int_{\partial \Omega}(\boldsymbol{x} \cdot \boldsymbol{n})\left|\epsilon^{1 / 2} \boldsymbol{E}_{N}\right|^{2} \mathrm{~d} S \\
& +\operatorname{Re}\left[\omega^{2} \int_{\partial \Omega} \epsilon(\boldsymbol{E} \cdot \boldsymbol{n})\left(\boldsymbol{x}_{T} \cdot \overline{\boldsymbol{E}}_{T}\right)\right] \\
\geq & -\frac{\omega^{2}}{2} \int_{\partial \Omega}(\boldsymbol{x} \cdot \boldsymbol{n})\left|\epsilon^{1 / 2} \boldsymbol{E}_{T}\right|^{2} \mathrm{~d} S-\frac{\omega^{2}}{2} \int_{\partial \Omega} \frac{1}{\boldsymbol{x} \cdot \boldsymbol{n}}\left|\boldsymbol{x}_{T}\right|^{2}\left|\epsilon^{1 / 2} \boldsymbol{E}_{T}\right|^{2} \mathrm{~d} S \\
= & -\frac{\omega^{2}}{2} \int_{\partial \Omega} \frac{|\boldsymbol{x}|^{2}}{\boldsymbol{x} \cdot \boldsymbol{n}}\left|\epsilon^{1 / 2} \boldsymbol{E}_{T}\right|^{2} \mathrm{~d} S .
\end{aligned}
$$


In order to estimate the term $T 2$, we replace $i \omega \lambda \boldsymbol{E}_{T}$ on $\partial \Omega$ by its expression given by the boundary condition, i.e.,

$$
i \omega \lambda \boldsymbol{E}_{T}=-\boldsymbol{n} \times\left(\mu^{-1} \nabla \times \boldsymbol{E}\right)-\boldsymbol{g} \quad \text { on } \partial \Omega,
$$

and get

$$
\begin{aligned}
T 2= & \frac{1}{2} \int_{\partial \Omega}(\boldsymbol{x} \cdot \boldsymbol{n})\left|\mu^{-1 / 2} \nabla \times \boldsymbol{E}\right|^{2} \mathrm{~d} S \\
& +\operatorname{Re}\left[\int_{\partial \Omega}\left[\boldsymbol{n} \times\left(\mu^{-1} \nabla \times \boldsymbol{E}\right)+\boldsymbol{g}\right] \cdot((\nabla \times \overline{\boldsymbol{E}}) \times \boldsymbol{x})_{T} \mathrm{~d} S\right] \\
= & -\frac{1}{2} \int_{\partial \Omega}(\boldsymbol{x} \cdot \boldsymbol{n})\left|\mu^{-1 / 2} \nabla \times \boldsymbol{E}\right|^{2} \mathrm{~d} S \\
& +\operatorname{Re}\left[\int_{\partial \Omega}\left(\left(\mu^{-1 / 2} \nabla \times \boldsymbol{E}\right) \cdot \boldsymbol{n}\right)\left(\left(\mu^{-1 / 2} \nabla \times \overline{\boldsymbol{E}}\right) \cdot \boldsymbol{x}\right) \mathrm{d} S\right] \\
& +\operatorname{Re}\left[\int_{\partial \Omega} \boldsymbol{g} \cdot((\nabla \times \overline{\boldsymbol{E}}) \times \boldsymbol{x})_{T} \mathrm{~d} S\right],
\end{aligned}
$$

where in the last step we have used $\boldsymbol{n} \times\left(\mu^{-1} \nabla \times \boldsymbol{E}\right) \cdot((\nabla \times \overline{\boldsymbol{E}}) \times \boldsymbol{x})_{T}=\boldsymbol{n} \times\left(\mu^{-1} \nabla \times\right.$ $\boldsymbol{E}) \cdot((\nabla \times \overline{\boldsymbol{E}}) \times \boldsymbol{x})$ and the identity

$$
(\boldsymbol{a} \times \boldsymbol{b}) \cdot(\boldsymbol{c} \times \boldsymbol{d})=(\boldsymbol{a} \cdot \boldsymbol{c})(\boldsymbol{b} \cdot \boldsymbol{d})-(\boldsymbol{b} \cdot \boldsymbol{c})(\boldsymbol{a} \cdot \boldsymbol{d}) .
$$

We proceed like in the estimate of $T 1$ and obtain

$$
T 2 \geq-\frac{1}{2} \int_{\partial \Omega} \frac{|\boldsymbol{x}|^{2}}{\boldsymbol{x} \cdot \boldsymbol{n}}\left|\left(\mu^{-1 / 2} \nabla \times \boldsymbol{E}\right)_{T}\right|^{2} \mathrm{~d} S+\operatorname{Re}\left[\int_{\partial \Omega} \boldsymbol{g} \cdot((\nabla \times \overline{\boldsymbol{E}}) \times \boldsymbol{x})_{T} \mathrm{~d} S\right] .
$$

By taking into account (2.3), (3.7) and the obtained estimates of $T 1$ and $T 2$, we obtain

$$
\begin{aligned}
& \frac{1}{2}\left\|\mu^{-1 / 2} \nabla \times \boldsymbol{E}\right\|_{0, \Omega}^{2}+\frac{\omega^{2}}{2}\left\|\epsilon^{1 / 2} \boldsymbol{E}\right\|_{0, \Omega}^{2} \\
& \quad-\frac{\omega^{2}}{2} \int_{\partial \Omega} \frac{|\boldsymbol{x}|^{2}}{\boldsymbol{x} \cdot \boldsymbol{n}}\left|\epsilon^{1 / 2} \boldsymbol{E}_{T}\right|^{2} \mathrm{~d} S-\frac{1}{2} \int_{\partial \Omega} \frac{|\boldsymbol{x}|^{2}}{\boldsymbol{x} \cdot \boldsymbol{n}}\left|\left(\mu^{-1 / 2} \nabla \times \boldsymbol{E}\right)_{T}\right|^{2} \mathrm{~d} S \\
& +\operatorname{Re}\left[\int_{\partial \Omega} \boldsymbol{g} \cdot((\nabla \times \overline{\boldsymbol{E}}) \times \boldsymbol{x})_{T} \mathrm{~d} S\right] \\
& \leq \operatorname{Re}\left[\int_{\Omega} \boldsymbol{J} \cdot((\nabla \times \overline{\boldsymbol{E}}) \times \boldsymbol{x}) \mathrm{d} V\right]+\operatorname{Re}\left[\int_{\partial \Omega} \boldsymbol{g} \cdot((\nabla \times \overline{\boldsymbol{E}}) \times \boldsymbol{x})_{T} \mathrm{~d} S\right],
\end{aligned}
$$


and thus, taking into account Lemma 3.1

$$
\begin{aligned}
& \left\|\mu^{-1 / 2} \nabla \times \boldsymbol{E}\right\|_{0, \Omega}^{2}+\omega^{2}\left\|\epsilon^{1 / 2} \boldsymbol{E}\right\|_{0, \Omega}^{2} \\
& \leq \omega^{2} \int_{\partial \Omega} \frac{|\boldsymbol{x}|^{2}}{\boldsymbol{x} \cdot \boldsymbol{n}}\left|\epsilon^{1 / 2} \boldsymbol{E}_{T}\right|^{2} \mathrm{~d} S+\int_{\partial \Omega} \frac{|\boldsymbol{x}|^{2}}{\boldsymbol{x} \cdot \boldsymbol{n}}\left|\left(\mu^{-1 / 2} \nabla \times \boldsymbol{E}\right)_{T}\right|^{2} \mathrm{~d} S \\
& \quad+2\left|\int_{\Omega} \boldsymbol{J} \cdot((\nabla \times \overline{\boldsymbol{E}}) \times \boldsymbol{x}) \mathrm{d} V\right| \\
& \leq \frac{d^{2}}{\gamma} \epsilon|\lambda|^{-1} \omega^{2}\left\|\lambda^{1 / 2} \boldsymbol{E}_{T}\right\|_{0, \partial \Omega}^{2}+\frac{d^{2}}{\gamma} \mu\left\|\left(\mu^{-1} \nabla \times \boldsymbol{E}\right)_{T}\right\|_{0, \partial \Omega}^{2} \\
& \quad+2\left|\int_{\Omega} \boldsymbol{J} \cdot((\nabla \times \overline{\boldsymbol{E}}) \times \boldsymbol{x}) \mathrm{d} V\right| .
\end{aligned}
$$

From (3.8) and (3.3), we have

$$
\begin{aligned}
\left\|\left(\mu^{-1} \nabla \times \boldsymbol{E}\right)_{T}\right\|_{0, \partial \Omega}^{2} & \leq 2|\lambda| \omega^{2}\left\|\lambda^{1 / 2} \boldsymbol{E}_{T}\right\|_{0, \partial \Omega}^{2}+2|\lambda|\left\|\lambda^{-1 / 2} \boldsymbol{g}\right\|_{0, \partial \Omega}^{2} \\
& \leq 4|\lambda| \omega\left|\int_{\Omega} \boldsymbol{J} \cdot \overline{\boldsymbol{E}} \mathrm{d} V\right|+4|\lambda|\left\|\lambda^{-1 / 2} \boldsymbol{g}\right\|_{0, \partial \Omega}^{2},
\end{aligned}
$$

which, together with (3.3), gives

$$
\begin{aligned}
\| \mu^{-1 / 2} \nabla & \times \boldsymbol{E}\left\|_{0, \Omega}^{2}+\omega^{2}\right\| \epsilon^{1 / 2} \boldsymbol{E} \|_{0, \Omega}^{2} \\
\leq & \frac{d^{2}}{\gamma}\left(\epsilon|\lambda|^{-1}+2 \mu|\lambda|\right) 2 \omega\left|\int_{\Omega} \boldsymbol{J} \cdot \overline{\boldsymbol{E}} \mathrm{d} V\right| \\
& +\frac{d^{2}}{\gamma}\left(\epsilon|\lambda|^{-1}+4 \mu|\lambda|\right)\left\|\lambda^{-1 / 2} \boldsymbol{g}\right\|_{0, \partial \Omega}^{2} \\
& +2\left|\int_{\Omega} \boldsymbol{J} \cdot((\nabla \times \overline{\boldsymbol{E}}) \times \boldsymbol{x}) \mathrm{d} V\right|
\end{aligned}
$$

Set, for convenience,

$$
Z:=\frac{d^{2}}{\gamma}\left(\epsilon|\lambda|^{-1}+4 \mu|\lambda|\right)
$$

the weighted Cauchy-Schwarz inequality gives

$$
\begin{aligned}
&\left\|\mu^{-1 / 2} \nabla \times \boldsymbol{E}\right\|_{0, \Omega}^{2}+\omega^{2}\left\|\epsilon^{1 / 2} \boldsymbol{E}\right\|_{0, \Omega}^{2} \\
& \leq Z\left(\frac{1}{\eta_{1}}\|\boldsymbol{J}\|_{0, \Omega}^{2}+\eta_{1} \epsilon^{-1} \omega^{2}\left\|\epsilon^{1 / 2} \boldsymbol{E}\right\|_{0, \Omega}^{2}\right) \\
&+Z\left\|\lambda^{-1 / 2} \boldsymbol{g}\right\|_{0, \partial \Omega}^{2}+\frac{1}{\eta_{2}}\|\boldsymbol{J}\|_{0, \Omega}^{2}+\eta_{2} d \mu\left\|\mu^{-1 / 2} \nabla \times \boldsymbol{E}\right\|_{0, \Omega}^{2} .
\end{aligned}
$$

We choose

$$
\eta_{1}=\frac{1}{2 Z \epsilon^{-1}}, \quad \eta_{2}=\frac{1}{2 d \mu}
$$


and obtain

$$
\begin{aligned}
& \frac{1}{2}\left\|\mu^{-1 / 2} \nabla \times \boldsymbol{E}\right\|_{0, \Omega}^{2}+\frac{1}{2} \omega^{2}\left\|\epsilon^{1 / 2} \boldsymbol{E}\right\|_{0, \Omega}^{2} \\
& \quad \leq\left(2 Z^{2} \epsilon^{-1}+2 d \mu\right)\|\boldsymbol{J}\|_{0, \Omega}^{2}+Z\left\|\lambda^{-1 / 2} \boldsymbol{g}\right\|_{0, \partial \Omega}^{2},
\end{aligned}
$$

i.e.,

$$
\begin{aligned}
& \left\|\mu^{-1 / 2} \nabla \times \boldsymbol{E}\right\|_{0, \Omega}+\omega\left\|\epsilon^{1 / 2} \boldsymbol{E}\right\|_{0, \Omega} \\
& \quad \leq\left(Z \epsilon^{-1 / 2}+d^{1 / 2} \mu^{1 / 2}\right)\|\boldsymbol{J}\|_{0, \Omega}+\left(Z|\lambda|^{-1}\right)^{1 / 2}\|\boldsymbol{g}\|_{0, \partial \Omega},
\end{aligned}
$$

from which gives the stability bound (3.1).

The bound (3.2) is obtained from (3.3) using the weighted Cauchy-Schwarz inequality and the bound (3.1).

The proof of the previous theorem hinges on the identities (3.5) and (3.6), obtained form the integration by parts of the two volume terms of the bilinear form $\mathcal{A}(\boldsymbol{E}, \boldsymbol{\xi})$ with the special test function $\boldsymbol{\xi}=(\nabla \times \boldsymbol{E}) \times \boldsymbol{x}$. These equalities are a generalization to the vector field setting of the so-called "Rellich-type identity" (see [23, Sect. 1.2 and Sect. 2], [6, Lemma 2.2, eq. (2.20)] and [7, Prop. 1]), and they have been used to prove analogous stability results, in the case of the Helmholtz equation, in $[7,11,17]$.

\subsection{Stability for polyhedral domains}

In order to prove the same result of Theorem 3.1 without assuming $\Omega$ to be of class $C^{2}$, we need to state some preliminary results.

Lemma 3.3. Let $\Omega \subset \mathbb{R}^{3}$ be a bounded polyhedral domain which is star-shaped with respect to $B_{\gamma}\left(\boldsymbol{x}_{0}\right)$, and let $R>0$ be such that $\bar{\Omega} \subset B_{R}\left(\boldsymbol{x}_{0}\right)$. Set $D:=B_{R}\left(\boldsymbol{x}_{0}\right) \backslash \Omega$. Then, if $z: \bar{D} \rightarrow \mathbb{R}$ is the continuous, harmonic function in $\Omega$, with $z=0$ on $\partial \Omega$ and $z=1$ on $\partial B_{R}\left(\boldsymbol{x}_{0}\right)$, then the radial derivative of $z$ is strictly positive at all points of $D$ and, for all $\delta \in(0,1)$, the domains

$$
\Omega_{\delta}:=\bar{\Omega} \cup\left\{\boldsymbol{x} \in \mathbb{R}^{3}: z(\boldsymbol{x})<\delta\right\}
$$

are $C^{\infty}$ and star-shaped with respect to $B_{\gamma}\left(\boldsymbol{x}_{0}\right)$. Moreover,

$$
\lim _{\delta \rightarrow 0} \operatorname{dist}\left(\partial \Omega, \partial \Omega_{\delta}\right)=0 .
$$

Proof. See [22, Thm. 2.2].

Lemma 3.4. Let $\Omega$ and $\Omega_{\delta}, 0<\delta<1$, be as in Lemma 3.3. Then, for every $\delta \in(0,1)$, there exists a homeomorphism $\Phi_{\delta}: \mathbb{R}^{3} \rightarrow \mathbb{R}^{3}$, bijective from $\Omega$ to $\Omega_{\delta}$, such that: 
i) there exists $\delta_{0} \in(0,1)$ such that

$$
\left\|D \Phi_{\delta}\right\|_{L^{\infty}(\bar{\Omega})^{3 x 3}}<C, \quad\left\|\left(D \Phi_{\delta}\right)^{-1}\right\|_{L^{\infty}(\bar{\Omega})^{3 x 3}}<C \quad \forall \delta \in\left(0, \delta_{0}\right),
$$

where $D \Phi_{\delta}$ is the Jacobian matrix of $\Phi_{\delta}$ and the constant $C>0$ is independent of $\delta$;

ii) denoting by $\operatorname{Id}_{3}$ the $3 \times 3$ identity matrix, it holds

$$
\lim _{\delta \rightarrow 0} D \Phi_{\delta}(\boldsymbol{x})=\lim _{\delta \rightarrow 0}\left(D \Phi_{\delta}(\boldsymbol{x})\right)^{-1}=\mathrm{Id}_{3} \quad \text { for a.e. } \boldsymbol{x} \in \bar{\Omega} \text { and a.e. } \boldsymbol{x} \in \partial \Omega .
$$

Proof. We assume, with no loss of generality, that $\boldsymbol{x}_{0}=\mathbf{0}$. For every point $\boldsymbol{x} \in$ $\mathbb{R}^{3} \backslash\{\mathbf{0}\}$ we denote its direction by $\hat{\boldsymbol{x}}=\boldsymbol{x} /|\boldsymbol{x}| \in \mathbb{S}^{2}$.

Since both $\Omega$ and $\Omega_{\delta}$ are open and star-shaped with respect to a neighborhood of $\mathbf{0}$, they can be described as follows:

$$
\begin{aligned}
\Omega & =\left\{\boldsymbol{x} \in \mathbb{R}^{3} \backslash\{\mathbf{0}\}:|\boldsymbol{x}|<\psi_{0}(\hat{\boldsymbol{x}})\right\} \cup\{\mathbf{0}\}, \\
\Omega_{\delta} & =\left\{\boldsymbol{x} \in \mathbb{R}^{3} \backslash\{\mathbf{0}\}:|\boldsymbol{x}|<\psi_{\delta}(\hat{\boldsymbol{x}})\right\} \cup\{\mathbf{0}\},
\end{aligned}
$$

where $\psi_{0} \in C^{0}\left(\mathbb{S}^{2}\right)$ and $\psi_{\delta} \in C^{\infty}\left(\mathbb{S}^{2}\right)$ are positive functions

$$
\psi_{0}, \psi_{\delta}: \mathbb{S}^{2} \rightarrow[\gamma, R]
$$

Notice that $\psi_{0}$ is piecewise $C^{\infty}$, but globally only continuous. (If $\Omega$ were star-shaped with respect to the origin only, then $\psi_{0}$ could be discontinuous.)

We define the homeomorphism $\Phi_{\delta}$ by

$$
\Phi_{\delta}(\boldsymbol{x})= \begin{cases}\frac{\psi_{\delta}(\hat{\boldsymbol{x}})}{\psi_{0}(\hat{\boldsymbol{x}})} \boldsymbol{x} & \boldsymbol{x} \in \mathbb{R}^{3} \backslash\{\mathbf{0}\}, \\ \mathbf{0} & \boldsymbol{x}=\mathbf{0} .\end{cases}
$$

This map is bijective and bicontinuous from $\mathbb{R}^{3}$ to itself, from $\Omega$ to $\Omega_{\delta}$ and from $\partial \Omega$ to $\partial \Omega_{\delta}$. Thanks to Lemma 3.3 we conclude that, for $\delta \rightarrow 0$,

$$
\psi_{\delta} \rightarrow \psi_{0} \quad \text { uniformly on } \mathbb{S}^{2},
$$

and, thus, $\Phi_{\delta}$ converges uniformly in $\bar{\Omega}$ to the identity.

In spherical coordinates $(r, \hat{\boldsymbol{x}}), \boldsymbol{x}=r \hat{\boldsymbol{x}}$, the mapping $\Phi_{\delta}$ reads

$$
\Phi_{\delta}(r, \hat{\boldsymbol{x}})=\left(\begin{array}{c}
\frac{\psi_{\delta}(\hat{\boldsymbol{x}})}{\psi_{0}(\hat{\boldsymbol{x}})} \\
\hat{\boldsymbol{x}}
\end{array}\right) .
$$

Hence, the expressions of the Jacobian of $\Phi_{\delta}$ and of its inverse in spherical coordinates are

$$
\begin{aligned}
D \Phi_{\delta}(\boldsymbol{x}) & =\left(\begin{array}{cc}
\psi_{\delta}(\hat{\boldsymbol{x}}) / \psi_{0}(\hat{\boldsymbol{x}}) & \frac{\partial}{\partial \hat{\boldsymbol{x}}}\left(\psi_{\delta}(\hat{\boldsymbol{x}}) / \psi_{0}(\hat{\boldsymbol{x}})\right) r \\
0 & \operatorname{Id}_{2}
\end{array}\right) \\
\left(D \Phi_{\delta}(\boldsymbol{x})\right)^{-1} & =\left(\begin{array}{cc}
\psi_{0}(\hat{\boldsymbol{x}}) / \psi_{\delta}(\hat{\boldsymbol{x}}) & -\psi_{0}(\hat{\boldsymbol{x}}) / \psi_{\delta}(\hat{\boldsymbol{x}}) \frac{\partial}{\partial \hat{\boldsymbol{x}}}\left(\psi_{\delta}(\hat{\boldsymbol{x}}) / \psi_{0}(\hat{\boldsymbol{x}})\right) r \\
0 & \operatorname{Id}_{2}
\end{array}\right)
\end{aligned}
$$

for $\boldsymbol{x} \in \Omega \backslash\{\boldsymbol{0}\}$, where $\frac{\partial}{\partial \hat{\boldsymbol{x}}}$ is the surface gradient on $\mathbb{S}^{2}$. 
Thanks to (3.9), we have the following uniform bounds (with respect to $\hat{\boldsymbol{x}}$ and $\delta)$ :

$$
\left|\frac{\psi_{\delta}(\hat{\boldsymbol{x}})}{\psi_{0}(\hat{\boldsymbol{x}})}\right| \leq \frac{R}{\gamma} \quad, \quad\left|\frac{\psi_{0}(\hat{\boldsymbol{x}})}{\psi_{\delta}(\hat{\boldsymbol{x}})}\right| \leq 1 \quad \forall \hat{\boldsymbol{x}} \in \mathbb{S}^{2}, \delta \in(0,1)
$$

therefore, in order to prove assertion $i$ ), we only need to prove a uniform bound on $\frac{\partial}{\partial \hat{\boldsymbol{x}}}\left(\psi_{\delta}(\hat{\boldsymbol{x}}) / \psi_{0}(\hat{\boldsymbol{x}})\right)$.

Temporarily, fix $\hat{\boldsymbol{x}} \in \mathbb{S}^{2}$ such that $\psi_{0}(\hat{\boldsymbol{x}}) \hat{\boldsymbol{x}}$ lies inside a face of $\Omega$. The surface gradient $\frac{\partial}{\partial \hat{\boldsymbol{x}}}\left(\psi_{\delta}(\hat{\boldsymbol{x}}) / \psi_{0}(\hat{\boldsymbol{x}})\right)$ lies in a plane $\Pi$ containing the origin and $\hat{\boldsymbol{x}}$. We call $\theta$ the angular polar coordinate in $\Pi$; then the derivative of the ratio is

$$
\begin{aligned}
\left|\frac{\partial}{\partial \hat{\boldsymbol{x}}} \frac{\psi_{\delta}(\hat{\boldsymbol{x}})}{\psi_{0}(\hat{\boldsymbol{x}})}\right|=\left|\frac{\partial}{\partial \theta} \frac{\psi_{\delta}(\hat{\boldsymbol{x}})}{\psi_{0}(\hat{\boldsymbol{x}})}\right| & =\left|\frac{\psi_{0}(\hat{\boldsymbol{x}}) \frac{\partial}{\partial \theta} \psi_{\delta}(\hat{\boldsymbol{x}})-\psi_{\delta}(\hat{\boldsymbol{x}}) \frac{\partial}{\partial \theta} \psi_{0}(\hat{\boldsymbol{x}})}{\psi_{0}^{2}(\hat{\boldsymbol{x}})}\right| \\
& =\left|\frac{\psi_{0}(\hat{\boldsymbol{x}}) \tan \alpha_{\delta}-\psi_{\delta}(\hat{\boldsymbol{x}}) \tan \alpha_{0}}{\psi_{0}^{2}(\hat{\boldsymbol{x}})}\right|,
\end{aligned}
$$

for every $\hat{\boldsymbol{x}} \in \mathbb{S}^{2}$ such that $\psi_{0}(\hat{\boldsymbol{x}}) \hat{\boldsymbol{x}}$ belongs to the interior of one of the face of the polyhedron $\Omega$. Here $\alpha_{\delta}$ is the (acute) angle between the tangent to the circle $\Pi \cap \psi_{\delta}(\hat{\boldsymbol{x}}) \mathbb{S}^{2}$ and the line tangent to $\Pi \cap \partial \Omega_{\delta}$ in the point $\psi_{\delta}(\hat{\boldsymbol{x}}) \hat{\boldsymbol{x}} ; \alpha_{0}$ is the analogous angle for $\Omega$, see Figure 2 (left).
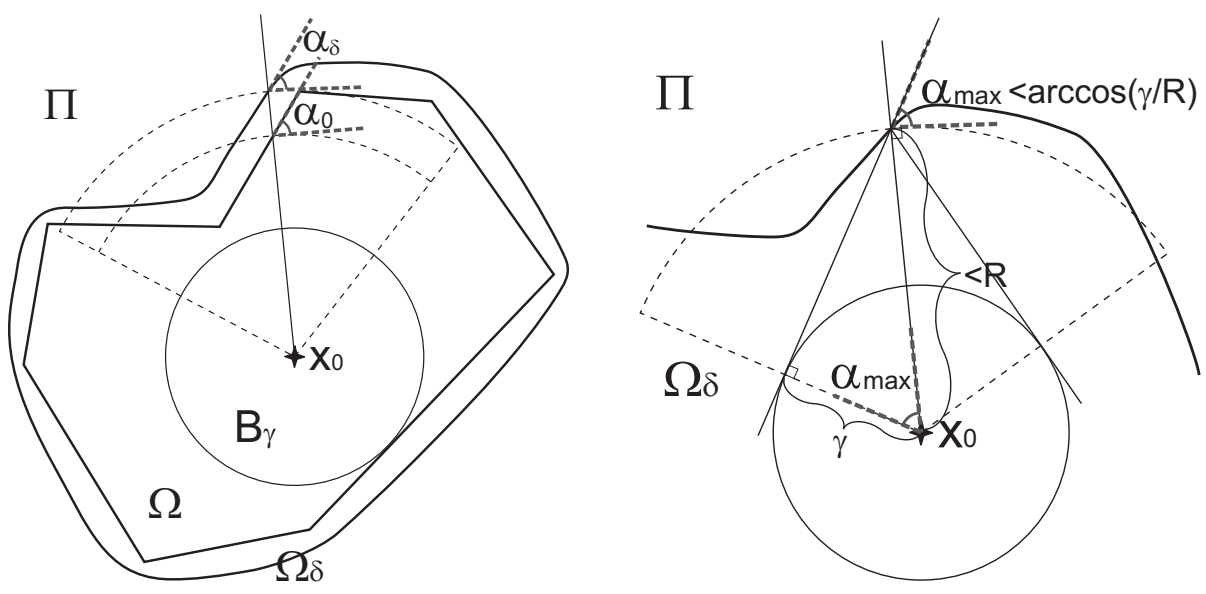

Fig. 2. A planar cross section of $\Omega$ and $\Omega_{\delta}$ with the angles $\alpha_{0}$ and $\alpha_{\delta}$ (left); the geometric argument that provides the upper bound on $\left|\alpha_{\delta}\right|$ (right).

Since $\Omega_{\delta}$ is star-shaped with respect to $B_{\gamma}(\mathbf{0})$ and it is contained in $B_{R}(\mathbf{0})$, $\left|\alpha_{\delta}\right|$ and $\left|\alpha_{0}\right|$ are bounded from above by $\arccos (\gamma / R)<\pi / 2$, as shown in Figure 2 (right). This implies that

$$
\tan \alpha_{\delta} \leq \tan \arccos (\gamma / R)=\frac{\sqrt{R^{2}-\gamma^{2}}}{\gamma}
$$


the same holds for $\alpha_{0}$. This in turns implies that the angular gradient of $\psi_{\delta}(\hat{\boldsymbol{x}}) / \psi_{0}(\hat{\boldsymbol{x}})$ is uniformly bounded, with respect to $\delta$ and $\hat{\boldsymbol{x}}$, for almost every $\hat{\boldsymbol{x}}$ (only the points corresponding to edges and vertices of $\Omega$ are excluded, because the gradient is not defined there). Finally, using the expression (3.10) of the Jacobians proves assertion $i$ ).

Let us consider $\boldsymbol{y} \in \partial \Omega$ such that it belongs to the interior of one of the faces of $\Omega$. Thanks to [2, Theorem 4.12], the harmonic function $z$ defined in Lemma 3.3 can be extended in a neighborhood of $\boldsymbol{y}$ to a harmonic function $z^{\prime}$. This implies that $z^{\prime}$ is $C^{\infty}$ in a neighborhood of $\boldsymbol{y}$.

Identifying $\Omega$ with $\Omega_{0}$, we notice that

$$
\partial \Omega_{\delta}=\left\{\boldsymbol{x} \in B_{R}(\mathbf{0}) \backslash \Omega: z^{\prime}(\boldsymbol{x})=\delta\right\}=\left\{\psi_{\delta}(\hat{\boldsymbol{x}}) \hat{\boldsymbol{x}}, \hat{\boldsymbol{x}} \in \mathbb{S}^{2}\right\} \quad \delta \in\left[0, \delta^{*}\right) .
$$

Thanks to the smoothness of $z^{\prime}$, we can apply the implicit function theorem (in polar coordinates) and prove that the function

$$
\begin{aligned}
\Psi: \mathbb{S}^{2} \times\left[0, \delta^{*}\right) & \rightarrow \mathbb{R} \\
(\hat{\boldsymbol{x}}, \delta) & \mapsto \psi_{\delta}(\hat{\boldsymbol{x}})
\end{aligned}
$$

is smooth in a neighborhood of $(\hat{\boldsymbol{y}}, 0)$. This implies the convergence

$$
\frac{\partial}{\partial \hat{\boldsymbol{y}}} \psi_{\delta}(\hat{\boldsymbol{y}}) \stackrel{\delta \rightarrow 0}{\longrightarrow} \frac{\partial}{\partial \hat{\boldsymbol{y}}} \psi_{0}(\hat{\boldsymbol{y}}) \quad \text { for a.e. } \hat{\boldsymbol{y}} \in \mathbb{S}^{2}
$$

We see from (3.10) and (3.11) that assertion ii) follows from this result and from the uniform convergence in $\mathbb{S}^{2}$ of $\psi_{\delta}$ to $\psi_{0}$.

We can now prove the main result of this section.

Theorem 3.2. Let $\Omega \subset \mathbb{R}^{3}$ be a bounded polyhedral domain which is star-shaped with respect to $B_{\gamma}\left(\boldsymbol{x}_{0}\right)$. Under the assumptions made on $\boldsymbol{J}, \boldsymbol{g}$ and on the material coefficients in Section [ 2 the result of Theorem 3.1 holds true.

Proof. Inspired by the proof of [10, Thm. 3.2.1.3], we harness the $\delta$-uniform stability result of Theorem 3.1 for the smooth domains $\Omega_{\delta}$ introduced in Lemma 3.3

Temporarily, fix $\delta \in(0,1)$. First we map the data $\boldsymbol{J}$ and $\boldsymbol{g}$ in (2.1) to $\Omega_{\delta}$ by suitable pullbacks w.r.t. $\Phi_{\delta}^{-1}$ : for almost all $\widetilde{\boldsymbol{x}}:=\Phi_{\delta}(\boldsymbol{x}) \in \Omega_{\delta}$ we define

$$
\begin{aligned}
\boldsymbol{J}_{\delta}(\widetilde{\boldsymbol{x}}) & :=(\operatorname{det} D \Phi(\boldsymbol{x}))^{-1} D \Phi(\boldsymbol{x}) \boldsymbol{J}(\boldsymbol{x}), \\
\boldsymbol{g}_{\delta}(\widetilde{\boldsymbol{x}}) & :=D \Phi(\boldsymbol{x})^{-T} \boldsymbol{g}(\boldsymbol{x}) .
\end{aligned}
$$

Note that this ensures $\widetilde{\nabla} \cdot \boldsymbol{J}_{\delta}=0$, if $\boldsymbol{J}$ is divergence frea. In addition, $\boldsymbol{g}_{\delta}$ will be a tangential vector field on $\partial \Omega_{\delta}$, provided that $\boldsymbol{g}$ is tangential to $\partial \Omega$, see the

a The operator $\widetilde{\nabla}$ indicates differentiation w.r.t. $\widetilde{\boldsymbol{x}} \in \Omega_{\delta}$. 
discussion of pullbacks in [12, Sect. 2.2]. From the defining formulas and Lemma 3.4 we immediately infer that, for all $0<\delta<\delta_{0}$,

$$
\begin{aligned}
& C^{-1}\|\boldsymbol{J}\|_{0, \Omega} \leq\left\|\boldsymbol{J}_{\delta}\right\|_{0, \Omega_{\delta}} \leq C\|\boldsymbol{J}\|_{0, \Omega}, \\
& C^{-1}\|\boldsymbol{g}\|_{0, \partial \Omega} \leq\left\|\boldsymbol{g}_{\delta}\right\|_{0, \partial \Omega_{\delta}} \leq C\|\boldsymbol{g}\|_{0, \partial \Omega} .
\end{aligned}
$$

Next, we introduce $\boldsymbol{E}_{\delta} \in H_{\mathrm{imp}}\left(\operatorname{curl} ; \Omega_{\delta}\right)$ as solution of the variational problem

$$
\mathcal{A}_{\delta}\left(\boldsymbol{E}_{\delta}, \boldsymbol{\xi}\right)=\int_{\Omega_{\delta}} \boldsymbol{J}_{\delta} \cdot \overline{\boldsymbol{\xi}} \mathrm{d} V+\int_{\partial \Omega_{\delta}} \boldsymbol{g}_{\delta} \cdot \overline{\boldsymbol{\xi}}_{T} \mathrm{~d} S \quad \forall \boldsymbol{\xi} \in H_{\mathrm{imp}}\left(\operatorname{curl} ; \Omega_{\delta}\right),
$$

where the bilinear form $\mathcal{A}_{\delta}(\cdot, \cdot)$ is the counterpart of $\mathcal{A}(\cdot, \cdot)$ from (2.3) in the domain $\Omega_{\delta}$. Theorem 2.1 guarantees existence and uniqueness of $\boldsymbol{E}_{\delta}$. Further, from Theorem 3.1] together with (3.12), we conclude the $\delta$-uniform bound

$$
\begin{array}{r}
\left\|\mu^{-1 / 2} \widetilde{\nabla} \times \boldsymbol{E}_{\delta}\right\|_{0, \Omega_{\delta}}+\omega\left\|\varepsilon^{1 / 2} \boldsymbol{E}_{\delta}\right\|_{0, \Omega_{\delta}}+\left\|\lambda^{1 / 2} \boldsymbol{E}_{\delta, T}\right\|_{0, \partial \Omega_{\delta}} \\
\leq C\left(\|\boldsymbol{J}\|_{0, \Omega}+\|\boldsymbol{g}\|_{0, \partial \Omega}\right),
\end{array}
$$

with a constant $C>0$ depending only on the coefficients $\varepsilon, \mu, \lambda$ and the geometry parameters $\gamma$ and $\operatorname{diam}(\Omega)$. Note that the $\delta$-uniform bound on $C$ is a consequence of the information on the shape of the $\Omega_{\delta}$ 's gleaned from Lemma 3.3

We pull $\boldsymbol{E}_{\delta}$ back to $\Omega$

$$
\widehat{\boldsymbol{E}}_{\delta}(\boldsymbol{x}):=D \Phi_{\delta}^{T}(\boldsymbol{x}) \boldsymbol{E}_{\delta}(\widetilde{\boldsymbol{x}}), \quad \text { a.e. } \boldsymbol{x} \in \Omega,
$$

with the special property $[12,(2.15)]$

$$
\left(\nabla \times \widehat{\boldsymbol{E}}_{\delta}\right)(\boldsymbol{x})=\operatorname{det} D \Phi_{\delta}(\boldsymbol{x}) D \Phi_{\delta}(\boldsymbol{x})^{-1}\left(\widetilde{\nabla} \times \boldsymbol{E}_{\delta}\right)(\widetilde{\boldsymbol{x}}), \quad \boldsymbol{x} \in \Omega .
$$

We easily find $\widehat{\boldsymbol{E}}_{\delta} \in H_{\mathrm{imp}}(\operatorname{curl} ; \Omega)$, since, thanks to Lemma 3.4 .

$$
\begin{array}{rlrl}
C^{-1}\left\|\widehat{\boldsymbol{E}}_{\delta}\right\|_{0, \Omega} & \leq\left\|\boldsymbol{E}_{\delta}\right\|_{0, \Omega_{\delta}} & \leq C\left\|\widehat{\boldsymbol{E}}_{\delta}\right\|_{0, \Omega} \\
C^{-1}\left\|\nabla \times \widehat{\boldsymbol{E}}_{\delta}\right\|_{0, \Omega} \leq\left\|\widetilde{\nabla} \times \boldsymbol{E}_{\delta}\right\|_{0, \Omega_{\delta}} & \leq C\left\|\nabla \times \widehat{\boldsymbol{E}}_{\delta}\right\|_{0, \Omega}, \\
C^{-1}\left\|\widehat{\boldsymbol{E}}_{\delta, T}\right\|_{0, \partial \Omega} \leq\left\|\boldsymbol{E}_{\delta}\right\|_{0, \partial \Omega_{\delta}} & \leq C\left\|\widehat{\boldsymbol{E}}_{\delta}\right\|_{0, \partial \Omega},
\end{array}
$$

for all $0<\delta<\delta_{0}$. We can even conclude a counterpart of (3.14)

$$
\begin{array}{r}
\left\|\mu^{-1 / 2} \nabla \times \widehat{\boldsymbol{E}}_{\delta}\right\|_{0, \Omega}+\omega\left\|\varepsilon^{1 / 2} \widehat{\boldsymbol{E}}_{\delta}\right\|_{0, \Omega}+\left\|\lambda^{1 / 2} \widehat{\boldsymbol{E}}_{\delta, T}\right\|_{0, \partial \Omega} \\
\leq C\left(\|\boldsymbol{J}\|_{0, \Omega}+\|\boldsymbol{g}\|_{0, \partial \Omega}\right) .
\end{array}
$$

Using (3.15) and (3.16) we can transform the variational equation (3.13) to $\Omega$ and find

$$
\widehat{\mathcal{A}}_{\delta}\left(\widehat{\boldsymbol{E}}_{\delta}, \widehat{\boldsymbol{\xi}}\right):=\int_{\Omega} \mu^{-1} \boldsymbol{A}_{\delta}(\boldsymbol{x})\left(\nabla \times \widehat{\boldsymbol{E}}_{\delta}\right)(\boldsymbol{x}) \cdot(\nabla \times \overline{\widehat{\boldsymbol{\xi}}})(\boldsymbol{x})
$$




$$
\begin{array}{r}
-\omega^{2} \varepsilon \boldsymbol{B}_{\delta}(\boldsymbol{x}) \widehat{\boldsymbol{E}}_{\delta}(\boldsymbol{x}) \cdot \overline{\widehat{\boldsymbol{\xi}}}(\boldsymbol{x}) \mathrm{d} V(\boldsymbol{x})-i \omega \int_{\partial \Omega} \lambda \boldsymbol{C}_{\delta}(\boldsymbol{x}) \widehat{\boldsymbol{E}}_{\delta, T}(\boldsymbol{x}) \cdot \overline{\boldsymbol{\xi}}_{T}(\boldsymbol{x}) \mathrm{d} S(\boldsymbol{x}) \\
=\mathcal{A}_{\delta}\left(\boldsymbol{E}_{\delta}, \boldsymbol{\xi}\right) \stackrel{\text { B.13) }}{=} \int_{\Omega} \boldsymbol{J}(\boldsymbol{x}) \cdot \overline{\widehat{\boldsymbol{\xi}}}(\boldsymbol{x}) \mathrm{d} V(\boldsymbol{x})+\int_{\partial \Omega} \boldsymbol{C}_{\delta}(\boldsymbol{x}) \boldsymbol{g}_{T}(\boldsymbol{x}) \cdot \overline{\widehat{\boldsymbol{\xi}}}_{T}(\boldsymbol{x}) \mathrm{d} S(\boldsymbol{x})
\end{array}
$$

with matrix-valued functions on $\Omega$ and $\partial \Omega$, respectively,

$$
\begin{aligned}
& \boldsymbol{A}_{\delta}(\boldsymbol{x}):=\left(\operatorname{det} D \Phi_{\delta}(\boldsymbol{x})\right)^{-1} D \Phi_{\delta}(\boldsymbol{x})^{T} D \Phi_{\delta}(\boldsymbol{x}) \quad \text { for almost all } \boldsymbol{x} \in \Omega, \\
& \boldsymbol{B}_{\delta}(\boldsymbol{x}):=\operatorname{det} D \Phi_{\delta}(\boldsymbol{x}) D \Phi_{\delta}(\boldsymbol{x})^{-1} D \Phi_{\delta}(\boldsymbol{x})^{-T} \text { for almost all } \boldsymbol{x} \in \Omega, \\
& \boldsymbol{C}_{\delta}(\boldsymbol{x}):=D \Phi_{\delta}(\boldsymbol{x})^{-1} D \Phi_{\delta}(\boldsymbol{x})^{-T} G_{\delta}(\boldsymbol{x}) \quad \text { for almost all } \boldsymbol{x} \in \partial \Omega,
\end{aligned}
$$

where $G_{\delta} \in L^{\infty}(\partial \Omega)$ is the Gram determinant $\left(\left\{\boldsymbol{t}_{1}, \boldsymbol{t}_{2}\right\}\right.$ is any orthonormal basis of the tangent space to $\partial \Omega$ at $\boldsymbol{x}$ )

$$
G_{\delta}(\boldsymbol{x})=\left|D \Phi_{\delta} \boldsymbol{t}_{1} \times D \Phi_{\delta}(\boldsymbol{x}) \boldsymbol{t}_{2}\right|, \quad \boldsymbol{x} \in \partial \Omega .
$$

We remark that, thanks to Lemma 3.4 part ii), the matrix functions $\boldsymbol{A}_{\delta}, \boldsymbol{B}_{\delta}$ and $\boldsymbol{C}_{\delta}$ are $L^{2}$-convergent to the $3 \times 3$ identity matrix $\operatorname{Id}_{3}$, for $\delta \rightarrow 0$. Eventually, in (3.18) the test function $\widehat{\boldsymbol{\xi}}$ is obtained from $\boldsymbol{\xi} \in H_{\mathrm{imp}}\left(\mathrm{curl} ; \Omega_{\delta}\right)$ by the transformation (3.15). However, since the transformation $H_{\mathrm{imp}}\left(\operatorname{curl} ; \Omega_{\delta}\right) \rightarrow H_{\mathrm{imp}}(\operatorname{curl} ; \Omega), \boldsymbol{\xi} \mapsto \widehat{\boldsymbol{\xi}}:=D \Phi_{\delta}^{T} \boldsymbol{\xi}$ is an isomorphism, (3.18) holds for any $\boldsymbol{\xi} \in H_{\mathrm{imp}}(\operatorname{curl} ; \Omega)$ in place of $\widehat{\xi}$.

Owing to (3.17), we can find a sequence $\left(\delta_{n}\right)_{n \in \mathbb{N}} \subset\left(0, \delta_{0}\right), \delta_{n} \rightarrow 0$ for $n \rightarrow \infty$, and a vector field $\boldsymbol{E}^{*} \in H_{\text {imp }}(\operatorname{curl} ; \Omega)$, such that, with $\widehat{\boldsymbol{E}}_{n}:=\widehat{\boldsymbol{E}}_{\delta_{n}}$,

$$
\widehat{\boldsymbol{E}}_{n} \stackrel{n \rightarrow \infty}{\longrightarrow} \boldsymbol{E}^{*} \quad \text { weakly in } \quad H_{\mathrm{imp}}(\operatorname{curl} ; \Omega) \text {. }
$$

Observe that the weak limit $\boldsymbol{E}^{*}$ also complies with the bound (3.17). To finish the proof, we have to show that $\boldsymbol{E}^{*}$ is the unique solution of the Maxwell variational problem (2.3).

The sequences $\left(\boldsymbol{A}_{\delta_{n}}\right)_{n \in \mathbb{N}},\left(\boldsymbol{B}_{\delta_{n}}\right)_{n \in \mathbb{N}},\left(\boldsymbol{C}_{\delta_{n}}\right)_{n \in \mathbb{N}}$ converge to $\operatorname{Id}_{3}$, for $n \rightarrow \infty$, in $L^{2}$ and almost everywhere in the respective domains; moreover, due to Lemma 3.4 part $i$ ), they are also uniformly bounded with respect to $\delta_{n}$. Since $\widehat{\boldsymbol{\xi}} \in H_{\mathrm{imp}}(\operatorname{curl} ; \Omega)$, by the dominated convergence theorem, we have

$$
\begin{aligned}
& \boldsymbol{A}_{\delta_{n}}^{T} \nabla \times \overline{\widehat{\boldsymbol{\xi}}} \stackrel{n \rightarrow \infty}{\longrightarrow} \nabla \times \overline{\widehat{\boldsymbol{\xi}}}, \\
& \boldsymbol{B}_{\delta_{n}}^{T} \overline{\widehat{\boldsymbol{\xi}}} \stackrel{n \rightarrow \infty}{\longrightarrow} \overline{\widehat{\boldsymbol{\xi}}}, \\
& C_{\delta_{n}}^{T} \overline{\boldsymbol{\xi}}_{T} \stackrel{n \rightarrow \infty}{\longrightarrow} \overline{\boldsymbol{\xi}}_{T},
\end{aligned}
$$

strongly in the sense of $L^{2}$. Consequently, since $\left(\widehat{\boldsymbol{E}}_{n}\right)_{n}$ is weakly convergent in 
$H_{\mathrm{imp}}(\operatorname{curl} ; \Omega)$, the weak versus strong convergence gives, for instance,

$$
\begin{aligned}
\int_{\Omega} & \mu^{-1} \boldsymbol{A}_{\delta_{n}}(\boldsymbol{x})\left(\nabla \times \widehat{\boldsymbol{E}}_{n}\right)(\boldsymbol{x}) \cdot(\nabla \times \overline{\widehat{\boldsymbol{\xi}}})(\boldsymbol{x}) \mathrm{d} V(\boldsymbol{x}) \\
= & \int_{\Omega} \underbrace{\left[\mu^{-1}\left(\nabla \times \widehat{\boldsymbol{E}}_{n}\right)(\boldsymbol{x})\right]}_{\text {weak }} \cdot \underbrace{\left[\boldsymbol{A}_{\delta_{n}}(\boldsymbol{x})^{T}(\nabla \times \overline{\boldsymbol{\xi}})(\boldsymbol{x})\right]}_{\text {strong }} \mathrm{d} V(\boldsymbol{x}) \\
& \stackrel{n \rightarrow \infty}{\longrightarrow} \int_{\Omega} \mu^{-1}\left(\nabla \times \boldsymbol{E}^{*}\right)(\boldsymbol{x}) \cdot(\nabla \times \overline{\boldsymbol{\xi}})(\boldsymbol{x}) \mathrm{d} V(\boldsymbol{x}) ;
\end{aligned}
$$

the other integrals in the definition of $\widehat{\mathcal{A}}_{\delta_{n}}\left(\widehat{\boldsymbol{E}}_{n}, \widehat{\boldsymbol{\xi}}\right)$ (see (3.18) ) are amenable to similar arguments, and, thus, we conclude

$$
\lim _{n \rightarrow \infty} \widehat{\mathcal{A}}_{\delta_{n}}\left(\widehat{\boldsymbol{E}}_{n}, \boldsymbol{\xi}\right)=\mathcal{A}\left(\boldsymbol{E}^{*}, \boldsymbol{\xi}\right) \quad \forall \boldsymbol{\xi} \in H_{\mathrm{imp}}(\operatorname{curl} ; \Omega) .
$$

Therefore,

$$
\begin{aligned}
\mathcal{A}\left(\boldsymbol{E}^{*}, \boldsymbol{\xi}\right) & \stackrel{\sqrt[3.18]{=}}{=} \lim _{n \rightarrow \infty}\left[\int_{\Omega} \boldsymbol{J}(\boldsymbol{x}) \cdot \overline{\boldsymbol{\xi}}(\boldsymbol{x}) \mathrm{d} V(\boldsymbol{x})+\int_{\partial \Omega} \boldsymbol{C}_{\delta_{n}}(\boldsymbol{x}) \boldsymbol{g}_{T}(\boldsymbol{x}) \cdot \overline{\boldsymbol{\xi}}_{T}(\boldsymbol{x}) \mathrm{d} S(\boldsymbol{x})\right] \\
= & \int_{\Omega} \boldsymbol{J}(\boldsymbol{x}) \cdot \overline{\boldsymbol{\xi}}(\boldsymbol{x}) \mathrm{d} V(\boldsymbol{x})+\int_{\partial \Omega} \boldsymbol{g}_{T}(\boldsymbol{x}) \cdot \overline{\boldsymbol{\xi}}_{T}(\boldsymbol{x}) \mathrm{d} S(\boldsymbol{x})
\end{aligned}
$$

by the dominated convergence theorem, because $\lim _{\delta \rightarrow 0} \boldsymbol{C}_{\delta}(\boldsymbol{x})=\operatorname{Id}_{3}$ a.e. on $\partial \Omega$, and $\left\|\boldsymbol{C}_{\delta}\right\|_{L^{\infty}(\partial \Omega)^{3 \times 3}}$ is $\delta$-uniformly bounded. From (3.19) and (3.17) we finally conclude the desired result.

\section{Regularity of solutions in polyhedral domains}

In this section, we establish the regularity of the solutions to problem (2.3) for polyhedral domains, when $\boldsymbol{g}$ possesses extra smoothness.

The definition of Sobolev spaces on the polyhedral boundary requires care. Denoting by $\Gamma_{j}, j=1, \ldots, m$, the flat faces of $\partial \Omega$, following [4, Section 2.3] we set

$$
H^{s}(\partial \Omega):= \begin{cases}\left\{\varphi \in H^{1}(\partial \Omega): \varphi_{\mid \Gamma_{j}} \in H^{s}\left(\Gamma_{j}\right), j=1, \ldots, m\right\} & \text { if } s \geq 1, \\ \left\{\varphi \in L^{2}(\partial \Omega): \varphi=\Phi_{\mid \partial \Omega} \text { for some } \Phi \in H^{s+1 / 2}(\Omega)\right\} & \text { if }|s|<1,\end{cases}
$$

and

$$
H_{T}^{s}(\partial \Omega):=\left\{\boldsymbol{\varphi} \in L_{T}^{2}(\partial \Omega): \boldsymbol{\varphi}_{\mid \Gamma_{j}} \in H^{s}\left(\Gamma_{j}\right)^{2}, j=1, \ldots, m\right\} \quad \forall s \geq 0 ;
$$


notice that in $[3,4]$ the space $H_{T}^{s}(\partial \Omega)$ was denoted $\boldsymbol{H}_{-}^{s}(\partial \Omega)$. The spaces $H^{s}(\partial \Omega)$ are endowed with the norms

$$
\|\varphi\|_{s, \partial \Omega}=\left\{\begin{array}{cl}
\left(\|\varphi\|_{1, \partial \Omega}^{2}+\sum_{j=1}^{m}\|\varphi\|_{s, \Gamma_{j}}^{2}\right)^{1 / 2} & \text { if } s \geq 1, \\
\inf _{\Phi \in H^{s+1 / 2}(\Omega): \Phi_{\mid \partial \Omega}=\varphi}\|\Phi\|_{s+1 / 2, \Omega} & \text { if }|s|<1 .
\end{array}\right.
$$

Thanks to Corollary 1.4.4.5 of [10] and the standard Sobolev trace theorem (see for instance [19, Thm. 3.9]), for $0<s<1 / 2$ the spaces $H^{s}(\partial \Omega)$ can be defined piecewise, i.e.,

$$
H^{s}(\partial \Omega)=\left\{\varphi \in L^{2}(\partial \Omega): \varphi_{\mid \Gamma_{j}} \in H^{s}\left(\Gamma_{j}\right), j=1, \ldots, m\right\}, \quad 0<s<1 / 2,
$$

with an equivalence between the two intrinsic norms; therefore we can identify the spaces

$$
H_{T}^{s}(\partial \Omega)=H^{s}(\partial \Omega)^{3} \cap L_{T}^{2}(\partial \Omega), \quad 0<s<1 / 2 .
$$

From [3, Thm. 3.9 and Thm. 3.10] (see also [5, Thm. 4.1]), we learn that, if $\boldsymbol{U} \in H(\operatorname{curl}, \Omega)$, then

$$
\begin{array}{r}
\operatorname{div}_{T}(\boldsymbol{U} \times \boldsymbol{n}) \in H^{-1 / 2}(\partial \Omega), \quad \operatorname{curl}_{T}\left(\boldsymbol{U}_{T}\right) \in H^{-1 / 2}(\partial \Omega), \\
\left\|\operatorname{div}_{T}(\boldsymbol{U} \times \boldsymbol{n})\right\|_{-1 / 2, \partial \Omega} \leq C\left(\|\boldsymbol{U}\|_{0, \Omega}+\|\nabla \times \boldsymbol{U}\|_{0, \Omega}\right), \\
\left\|\operatorname{curl}_{T}\left(\boldsymbol{U}_{T}\right)\right\|_{-1 / 2, \partial \Omega} \leq C\left(\|\boldsymbol{U}\|_{0, \Omega}+\|\nabla \times \boldsymbol{U}\|_{0, \Omega}\right),
\end{array}
$$

where $\operatorname{curl}_{T}$ and $\operatorname{div}_{T}$ are the surface curl and the surface divergence on $\partial \Omega$, respectively, and the constant $C>0$ is independent of $\boldsymbol{U}$.

The identifications (4.3) and (4.2) imply the continuity of the surface differential operators:

$$
\operatorname{div}_{T}, \operatorname{curl}_{T}: H_{T}^{s}(\partial \Omega) \rightarrow H^{s-1}(\partial \Omega), \quad 0<s<1 / 2 .
$$

Eventually, the standard trace theorem for Sobolev spaces yields the continuity of the tangential traces (see [3, p. 11])

$$
\left\{\begin{array}{rl}
H^{1}(\Omega)^{3} & \rightarrow H_{T}^{1 / 2}(\partial \Omega) \\
\boldsymbol{U} & \mapsto \boldsymbol{U}_{T}
\end{array}, \quad\left\{\begin{array}{rl}
H^{1}(\Omega)^{3} & \rightarrow H_{T}^{1 / 2}(\partial \Omega) \\
\boldsymbol{U} & \mapsto \boldsymbol{U} \times \boldsymbol{n}
\end{array} .\right.\right.
$$

The following lemma provides a simple regularity result for the Laplace equation in the context of the spaces defined in (4.1). As a by-product, we obtain embeddings between the Sobolev spaces on $\partial \Omega$ defined piecewise as above and the ones defined as traces of functions in $\Omega$, as in [19]; see Remark 4.2 below.

Lemma 4.1. Let $\Omega$ be a Lipschitz polyhedron. Then there exists $s_{\Omega}$ depending only on $\Omega, 0<s_{\Omega}<1 / 2$, such that if $\varphi$ satisfies

$$
\left\{\begin{aligned}
-\Delta \varphi=0 & \text { on } \Omega, \\
\varphi_{\mid \partial \Omega} & \in H^{s}(\partial \Omega),
\end{aligned}\right.
$$


for some $1<s \leq 1+s_{\Omega}$, then $\varphi$ belongs to $H^{s+1 / 2}(\Omega)$. Moreover the following bound holds

$$
\|\varphi\|_{s+1 / 2, \Omega} \leq C\|\varphi\|_{s, \partial \Omega} .
$$

Proof. We know from the definition (4.1) that the trace operator from $H^{t}(\Omega)$ to $H^{t-1 / 2}(\partial \Omega)$ is continuous and surjective for any $1 / 2<t<1$. This, together with (4.3), implies that Proposition 3.7 in [1] (see also [19, Thm. 3.50]) can be slightly generalized as follows: given

$$
\boldsymbol{v} \in H(\operatorname{curl} ; \Omega) \cap H(\operatorname{div} ; \Omega) \quad \text { such that } \quad \boldsymbol{v}_{T} \in H_{T}^{t-1 / 2}(\partial \Omega), \quad 1 / 2<t<1,
$$

then $\boldsymbol{v} \in H^{\min \left\{1 / 2+s_{\Omega}, t\right\}}(\Omega)$ for some positive $s_{\Omega}$ depending only on $\Omega$. Also the bound in [19, Thm. 3.50] can be generalized as

$$
\|\boldsymbol{v}\|_{t, \Omega} \leq C\left(\|\boldsymbol{v}\|_{0, \Omega}+\|\nabla \times \boldsymbol{v}\|_{0, \Omega}+\|\nabla \cdot \boldsymbol{v}\|_{0, \Omega}+\|\boldsymbol{v}\|_{t-1 / 2, \partial \Omega}\right),
$$

for every $1 / 2<t \leq 1 / 2+s_{\Omega}<1$.

We define the vector field $\boldsymbol{w}=\nabla \varphi$, it satisfies the condition 4.8 since $\nabla \times \boldsymbol{w}=\mathbf{0}, \nabla \cdot \boldsymbol{w}=0$ and its tangential trace is $\boldsymbol{w}_{T}=\nabla_{T} \varphi \in H^{s-1}(\partial \Omega)^{3} \cap$ $L^{2}(\partial \Omega)=H_{T}^{s-1}(\partial \Omega)$ thanks to 4.3); therefore $\boldsymbol{w}$ belongs to $H^{s-1 / 2}(\Omega)$ and finally $\varphi \in H^{s+1 / 2}(\Omega)$.

The bound (4.7) follows from

$$
\begin{aligned}
\|\varphi\|_{s+1 / 2, \Omega} & \leq C\left(\|\varphi\|_{0, \Omega}+\|\boldsymbol{w}\|_{s-1 / 2, \Omega}\right) \\
& \stackrel{4.9}{\leq} C\left(\|\varphi\|_{0, \Omega}+\|\boldsymbol{w}\|_{0, \Omega}+\left\|\nabla_{T} \varphi\right\|_{s-1, \partial \Omega}\right) \\
& \stackrel{4.2]}{\leq} C\left(\|\varphi\|_{1 / 2, \partial \Omega}+\sum_{j=1}^{m}\left\|\nabla_{T} \varphi\right\|_{s-1, \Gamma_{j}}\right) \\
& \leq C\|\varphi\|_{s, \partial \Omega},
\end{aligned}
$$

where we have used the usual $H^{1}$-stability of the Laplace problem for $u$, see for instance [19, Thm. 3.12].

Remark 4.1. From the proof of the previous lemma, it is clear that the parameter $s_{\Omega}$ is equal to the one called $s$ in [1, Prop. 3.7].

Whenever the domain is convex, Theorem 2.17 of [1] applies and Lemma 4.1 holds for every $1<s<3 / 2$.

Remark 4.2. Lemma 4.1 provides the embedding

$$
H^{s}(\partial \Omega) \subseteq\left\{\varphi \in L^{2}(\partial \Omega): \varphi=\Phi_{\mid \partial \Omega} \text { for some } \Phi \in H^{s+1 / 2}(\Omega)\right\},
$$

for $1<s<1+s_{\Omega}$; moreover the immersion is continuous, i.e.,

$$
\inf _{\Phi \in H^{s+1 / 2}(\Omega), \Phi_{\mid \partial \Omega}=\varphi}\|\Phi\|_{s+1 / 2, \Omega} \leq C\|\varphi\|_{s, \partial \Omega} .
$$


The opposite inclusion

$$
H^{s}(\partial \Omega) \supseteq\left\{\varphi \in L^{2}(\partial \Omega): \varphi=\Phi_{\mid \partial \Omega} \text { for some } \Phi \in H^{s+1 / 2}(\Omega)\right\}
$$

holds in the larger range $1<s<3 / 2$ and it is a simple consequence of the definition (4.1): for every $\Phi \in H^{s+1 / 2}(\Omega), 1<s<3 / 2$,

$$
\begin{aligned}
\|\Phi\|_{s, \partial \Omega} & \leq C\left(\|\Phi\|_{0, \partial \Omega}+\|\nabla \Phi\|_{0, \partial \Omega}+\sum_{j=1}^{m}\|\nabla \Phi\|_{s-1, \Gamma_{j}}\right) \\
& \stackrel{4.2]}{\leq} C\left(\|\Phi\|_{0, \partial \Omega}+\|\nabla \Phi\|_{s-1, \partial \Omega}\right) \\
& \leq C\|\Phi\|_{s+1 / 2, \Omega} .
\end{aligned}
$$

Notice that in the limit case $s=3 / 2$, 44.10) does not hold: Theorem 3.4 of [3] shows that the traces of functions in $H^{2}(\Omega)$ can not be defined piecewise on the faces of a polyhedron, but tangential continuity is required along the edges.

Remark 4.3. Lemma (4.1) implies Theorem 3.18 of [19]. Notice that the definition of $H^{s}(\partial \Omega)$ for $s>1$ given in [19, (3.12)] is different from the one used in this paper.

A last elliptic regularity result will be instrumental in the treatment of Maxwell solutions: it concerns the Laplace-Beltrami operator $\Delta_{T}=\operatorname{div}_{T} \nabla_{T}$, where $\nabla_{T}$ denotes the tangential gradient, and is stated in [4, Thm. 8]; we report it here, for the sake of completeness.

Lemma 4.2. For any bounded Lipschitz polyhedral domain, there is a $0<s^{*} \leq 1$ depending only on the shape of $\partial \Omega$ in neighborhoods of vertices, such that

$$
\begin{gathered}
\Delta_{T} \psi \in H^{-1+s}(\partial \Omega) \text { for some } s>0 \\
\Rightarrow \quad \psi \in H^{1+s_{L B}}(\partial \Omega) \quad \forall 0<s_{L B} \leq s, s_{L B}<s^{*} .
\end{gathered}
$$

The case $s_{L B}=s$, when $s<s^{*}$, can be deduced from the proof of [4, Thm. 8]. Moreover, formula (57) in [4] shows that, whenever $\Omega$ is convex, it is possible to choose $s^{*}=1$.

We are now ready to prove the main theorem of this section, namely, a regularity result for the solutions of the Maxwell equations.

Theorem 4.4. Let $\Omega \subset \mathbb{R}^{3}$ be a bounded polyhedral domain which is star-shaped with respect to $B_{\gamma}\left(\boldsymbol{x}_{0}\right)$. In addition to the assumptions made on $\boldsymbol{J}, \boldsymbol{g}$ and on the material coefficients in Section $\mathbf{Q}$, we assume $\boldsymbol{g} \in H_{T}^{s_{g}}(\partial \Omega)$, with $0<s_{g}<1 / 2$. Then the solution $\boldsymbol{E}$ to problem (2.3) satisfies

$$
\boldsymbol{E} \in H^{1 / 2+s}(\Omega)^{3} \quad \text { and } \quad \nabla \times \boldsymbol{E} \in H^{1 / 2+s}(\Omega)^{3}
$$

for all the real parameters $s$ such that

$$
0<s \leq \min \left\{s_{g}, s_{\Omega}\right\} \quad \text { and } s<s^{*},
$$

where $s_{\Omega}$ is defined in Lemma [4.1 (or in [1, Prop. 3.7]), and $s^{*}$ is defined in Lemma 4.2 (or in [4, Thm. 8]). 
Moreover, we have the following stability estimate: there is a positive constant $C$ independent of $\omega$, but depending on $s, \Omega, \gamma, \lambda, \epsilon$ and $\mu$, such that

$$
\|\nabla \times \boldsymbol{E}\|_{1 / 2+s, \Omega}+\omega\|\boldsymbol{E}\|_{1 / 2+s, \Omega} \leq C\left((1+\omega)\left(\|\boldsymbol{J}\|_{0, \Omega}+\|\boldsymbol{g}\|_{0, \partial \Omega}\right)+\|\boldsymbol{g}\|_{s_{g}, \partial \Omega}\right) .
$$

Proof. In this proof, we denote by $C$ a positive constant independent of $\omega$, but depending on $\lambda, \Omega, \epsilon$ and $\mu$, whose value might change at each occurrence.

We start by by proving the regularity of $\boldsymbol{E}$, following the reasoning of [8, Sect. 4.5.d].

Decompose $\boldsymbol{E}$ as

$$
\boldsymbol{E}=\boldsymbol{\Phi}^{0}+\nabla \psi
$$

where $\boldsymbol{\Phi}^{0} \in H^{1}(\Omega)^{3} \cap H\left(\operatorname{div}^{0} ; \Omega\right), \psi \in H^{1}(\Omega)$ and

$$
\left\|\boldsymbol{\Phi}^{0}\right\|_{1, \Omega}+\|\psi\|_{1, \Omega} \leq C\left(\|\boldsymbol{E}\|_{0, \Omega}+\|\nabla \times \boldsymbol{E}\|_{0, \Omega}\right)
$$

(see [12, Lemma 2.4]); clearly, $\Delta \psi=0$ in $\Omega$.

By using this decomposition, we can write the boundary condition in problem (2.2) by

$$
\left(\mu^{-1} \nabla \times \boldsymbol{E}\right) \times \boldsymbol{n}-i \omega \lambda \boldsymbol{\Phi}_{T}^{0}-i \omega \lambda \nabla_{T} \psi=\boldsymbol{g} \quad \text { on } \partial \Omega,
$$

where $\nabla_{T} \psi$ is the tangential gradient of $\psi$ on $\partial \Omega$, i.e., $\nabla_{T} \psi:=(\boldsymbol{n} \times \nabla \psi) \times \boldsymbol{n}$.

Using (4.4), the tangential divergence $\operatorname{div}_{T}$ of $\left(\mu^{-1} \nabla \times \boldsymbol{E}\right) \times \boldsymbol{n}$ is well-defined, belongs to $H^{-1 / 2}(\partial \Omega)$ and

$$
\begin{aligned}
\left\|\operatorname{div}_{T}\left(\left(\mu^{-1} \nabla \times \boldsymbol{E}\right) \times \boldsymbol{n}\right)\right\|_{-1 / 2, \partial \Omega} \\
\quad \leq C\left(\left\|\mu^{-1} \nabla \times \boldsymbol{E}\right\|_{0, \Omega}+\left\|\nabla \times\left(\mu^{-1} \nabla \times \boldsymbol{E}\right)\right\|_{0, \Omega}\right) .
\end{aligned}
$$

Since $\boldsymbol{g} \in H_{T}^{s_{g}}(\partial \Omega)$, (4.5) gives $\operatorname{div}_{T} \boldsymbol{g} \in H^{s_{g}-1}(\partial \Omega)$. Moreover, (4.6) and (4.5) imply $\operatorname{div}_{T} \boldsymbol{\Phi}_{T}^{0} \in H^{-1 / 2-\eta}(\partial \Omega)$ for all $\eta \in(0,1 / 2]$, in particular, $\operatorname{div}_{T} \boldsymbol{\Phi}_{T}^{0} \in H^{s_{g}-1}(\partial \Omega)$; they also imply the bounds

$$
\left\|\operatorname{div}_{T} \boldsymbol{\Phi}_{T}^{0}\right\|_{s_{g}-1, \partial \Omega} \leq C\left\|\boldsymbol{\Phi}_{T}^{0}\right\|_{s_{g}-1, \partial \Omega} \leq C\left\|\boldsymbol{\Phi}^{0}\right\|_{1, \partial \Omega} .
$$

From the regularities of the tangential divergence of the terms in (4.13), it follows that

$$
\operatorname{div}_{T} \lambda \nabla_{T} \psi \in H^{s_{g}-1}(\partial \Omega) .
$$

Due to the smoothness of the solutions to the Laplace-Beltrami equation provided by Lemma 4.2 we have that $\psi \in H^{1+s_{L B}}(\partial \Omega)$, for every $0<s_{L B} \leq s_{g}, s_{L B}<s^{*}$, where $s^{*}$ is defined in Lemma 4.2 Lemma 4.1 ensures that $\psi \in H^{3 / 2+s}(\Omega)$, for every $0<s \leq \min \left\{s_{g}, s_{\Omega}\right\}, s<s^{*}$, where $0<s_{\Omega}<1 / 2$ is given in Lemma 4.1].

\footnotetext{
${ }^{\mathrm{b}}$ Whenever $\Omega$ is convex, the parameter $L$ in [4, Thm. 8] is equal to $2 \pi$, thus $s^{*}=1$. Moreover, thanks to Remark 4.1 $s_{\Omega}$ can be chosen equal to $s_{g}$. Therefore, if $\Omega$ is convex, the only condition on $s$ is $0<s \leq s_{g}$.
} 
Moreover the previous steps give

$$
\|\psi\|_{3 / 2+s, \Omega} \stackrel{4.7}{\leq} C\|\psi\|_{1+s, \partial \Omega} \stackrel{[15, \text { eq. (2.2)] }}{\leq} C\left\|\operatorname{div}_{T} \lambda \nabla_{T} \psi\right\|_{s_{g}-1, \partial \Omega} .
$$

From $\boldsymbol{\Phi}^{0} \in H^{1}(\Omega)^{3}$ and $\nabla \psi \in H^{1 / 2+s}(\Omega)^{3}$, we have that $\boldsymbol{E} \in H^{1 / 2+s}(\Omega)^{3}$.

We proceed by bounding $\|\boldsymbol{E}\|_{1 / 2+s, \Omega}$. By the triangle inequality, we have

$$
\|\boldsymbol{E}\|_{1 / 2+s, \Omega} \leq\left\|\boldsymbol{\Phi}^{0}\right\|_{1 / 2+s, \Omega}+\|\nabla \psi\|_{1 / 2+s, \Omega},
$$

and we bound the two terms on the right-hand side separately.

From (4.12) and Theorem 3.2 (see (3.1)), we obtain

$$
\left\|\boldsymbol{\Phi}^{0}\right\|_{1, \Omega} \leq C\left(1+\omega^{-1}\right)\left(C_{1}\|\boldsymbol{J}\|_{0, \Omega}+C_{2}\|\boldsymbol{g}\|_{0, \partial \Omega}\right) .
$$

Collecting the bounds proved so far, we obtain

$$
\begin{aligned}
& \|\nabla \psi\|_{1 / 2+s, \Omega} \stackrel{4.15}{\leq} C\left\|\operatorname{div}_{T} \lambda \nabla_{T} \psi\right\|_{s_{g}-1, \partial \Omega} \\
& \stackrel{4.13}{\leq} C\left(\omega^{-1}\left\|\operatorname{div}_{T}\left(\left(\mu^{-1} \nabla \times \boldsymbol{E}\right) \times \boldsymbol{n}\right)\right\|_{s_{g}-1, \partial \Omega}\right. \\
& \left.+\left\|\operatorname{div}_{T} \lambda \boldsymbol{\Phi}_{T}^{0}\right\|_{s_{g}-1, \partial \Omega}+\omega^{-1}\left\|\operatorname{div}_{T} \boldsymbol{g}\right\|_{s_{g}-1, \partial \Omega}\right) \\
& \stackrel{4.5}{\leq} C\left(\omega^{-1}\left\|\operatorname{div}_{T}\left(\left(\mu^{-1} \nabla \times \boldsymbol{E}\right) \times \boldsymbol{n}\right)\right\|_{-1 / 2, \partial \Omega}\right. \\
& \left.+\left\|\Phi_{T}^{0}\right\|_{s_{g}, \partial \Omega}+\omega^{-1}\|\boldsymbol{g}\|_{s_{g}, \partial \Omega}\right) \\
& \stackrel{4.14,}{\leq} C\left(\omega^{-1}\|\nabla \times \boldsymbol{E}\|_{0, \Omega}+\omega^{-1}\|\nabla \times \nabla \times \boldsymbol{E}\|_{0, \Omega}\right. \\
& \left.+\left\|\Phi^{0}\right\|_{1, \Omega}+\omega^{-1}\|\boldsymbol{g}\|_{s_{g}, \partial \Omega}\right) \\
& \stackrel{2.22,4.16}{\leq} C\left(\omega^{-1}\|\nabla \times \boldsymbol{E}\|_{0, \Omega}+\omega\|\boldsymbol{E}\|_{0, \Omega}+\omega^{-1}\|\boldsymbol{J}\|_{0, \Omega}\right. \\
& \left.+\left(1+\omega^{-1}\right)\left(C_{1}\|\boldsymbol{J}\|_{0, \Omega}+C_{2}\|\boldsymbol{g}\|_{0, \partial \Omega}\right)+\omega^{-1}\|\boldsymbol{g}\|_{s_{g}, \partial \Omega}\right) \\
& \underset{\leq}{\text { 3.1], Thm. B.2] }} C\left(\left(C_{1}+\omega^{-1} C_{1}+\omega^{-1}\right)\|\boldsymbol{J}\|_{0, \Omega}\right. \\
& \left.+\left(1+\omega^{-1}\right) C_{2}\|\boldsymbol{g}\|_{0, \partial \Omega}+\omega^{-1}\|\boldsymbol{g}\|_{s_{g}, \partial \Omega}\right) .
\end{aligned}
$$

Therefore, we have the bound

$$
\omega\|\boldsymbol{E}\|_{1 / 2+s, \Omega} \leq C\left(\left(1+C_{1}+C_{1} \omega\right)\|\boldsymbol{J}\|_{0, \Omega}+(1+\omega) C_{2}\|\boldsymbol{g}\|_{0, \partial \Omega}+\|\boldsymbol{g}\|_{s_{g}, \partial \Omega}\right) .
$$

Similarly, we prove the smoothness of $\nabla \times \boldsymbol{E}$. Decompose $\nabla \times \boldsymbol{E}$ as

$$
\nabla \times \boldsymbol{E}=\boldsymbol{\Psi}^{0}+\nabla \phi,
$$

where $\boldsymbol{\Psi}^{0} \in H^{1}(\Omega)^{3} \cap H\left(\operatorname{div}^{0} ; \Omega\right)$, and $\phi \in H^{1}(\Omega)$; again, $\Delta \phi=0$ in $\Omega$ and

$$
\begin{aligned}
\left\|\Psi^{0}\right\|_{1, \Omega}+\|\phi\|_{1, \Omega} & \leq C\left(\|\nabla \times \boldsymbol{E}\|_{0, \Omega}+\|\nabla \times \nabla \times \boldsymbol{E}\|_{0, \Omega}\right) \\
& \leq C\left(\|\nabla \times \boldsymbol{E}\|_{0, \Omega}+\omega^{2}\|\boldsymbol{E}\|_{0, \Omega}+\|\boldsymbol{J}\|_{0, \Omega}\right),
\end{aligned}
$$


where the second inequality follows from the first equation in (2.2). The boundary condition in problem (2.2) can be written as

$$
\mu^{-1} \boldsymbol{\Psi}^{0} \times \boldsymbol{n}+\mu^{-1} \nabla \phi \times \boldsymbol{n}-i \omega \lambda \boldsymbol{E}_{T}=\boldsymbol{g} \quad \text { on } \partial \Omega .
$$

Thanks to (4.4), the tangential curl $\operatorname{curl}_{T}$ of $\lambda \boldsymbol{E}_{T}$ is well-defined, belongs to $H^{-1 / 2}(\partial \Omega)$ and

$$
\left\|\operatorname{curl}_{T} \lambda \boldsymbol{E}_{T}\right\|_{-1 / 2, \partial \Omega} \leq C\left(\|\boldsymbol{E}\|_{0, \Omega}+\|\nabla \times \boldsymbol{E}\|_{0, \Omega}\right) .
$$

Since $\boldsymbol{g} \in H_{T}^{s_{g}}(\partial \Omega)^{3}$, (4.5) gives $\operatorname{curl}_{T} \boldsymbol{g} \in H^{s_{g}-1}(\partial \Omega)$. Moreover, $\boldsymbol{\Psi}^{0} \times \boldsymbol{n} \in$ $H_{T}^{1 / 2}(\partial \Omega)^{3}$ by (4.6), then $\operatorname{curl}_{T}\left(\mu^{-1} \boldsymbol{\Psi}^{0} \times \boldsymbol{n}\right) \in H^{-1 / 2-\eta}(\partial \Omega)$, for every $0<\eta<1 / 2$, by (4.5), in particular, $\operatorname{curl}_{T}\left(\mu^{-1} \boldsymbol{\Psi}^{0} \times \boldsymbol{n}\right) \in H^{s_{g}-1}(\partial \Omega)$. Thus, since

$$
\operatorname{curl}_{T}\left(\mu^{-1} \nabla \phi \times \boldsymbol{n}\right)=-\operatorname{div}_{T}\left(\boldsymbol{n} \times\left(\mu^{-1} \nabla \phi \times \boldsymbol{n}\right)\right)=-\operatorname{div}_{T} \mu^{-1} \nabla_{T} \phi
$$

(see [18, Formula (3.15), p. 49]), we have that

$$
\operatorname{div}_{T} \mu^{-1} \nabla_{T} \phi \in H^{s_{g}-1}(\partial \Omega) .
$$

Proceeding exactly as we did to prove (4.15), Lemmas 4.1 and 4.2 ensure that the harmonic function $\phi$ belongs to $H^{3 / 2+s}(\Omega)$ with the parameter $s$ in the same range as before $\left(0<s \leq \min \left\{s_{g}, s_{\Omega}\right\}, s<s^{*}\right)$, and

$$
\|\phi\|_{3 / 2+s, \Omega} \stackrel{4.7}{\leq} C\|\phi\|_{1+s, \partial \Omega} \stackrel{[15, \text { eq. (2.2)] }}{\leq} C\left\|\operatorname{div}_{T} \lambda \nabla_{T} \phi\right\|_{s_{g}-1, \partial \Omega} .
$$

From $\Psi^{0} \in H^{1}(\Omega)^{3}$ and $\nabla \phi \in H^{1 / 2+s}(\Omega)^{3}$, we have that $\nabla \times \boldsymbol{E} \in H^{1 / 2+s}(\Omega)^{3}$.

For the bound of $\|\nabla \times \boldsymbol{E}\|_{1 / 2+s, \Omega}$, the triangle inequality gives

$$
\|\nabla \times \boldsymbol{E}\|_{1 / 2+s, \Omega} \leq\left\|\Psi^{0}\right\|_{1 / 2+s, \Omega}+\|\nabla \phi\|_{1 / 2+s, \Omega} .
$$

Again as in the first part of this proof, from (4.18) and Theorem 3.2 (see (3.1)), we have

$$
\left\|\Psi^{0}\right\|_{1, \Omega} \leq C\left(\left(1+C_{1}+C_{1} \omega\right)\|\boldsymbol{J}\|_{0, \Omega}+(1+\omega) C_{2}\|\boldsymbol{g}\|_{0, \partial \Omega}\right) .
$$

For $\|\nabla \phi\|_{1 / 2+s, \Omega}$, by proceeding as in the first part of this proof, using (4.21), the boundary condition (4.19), the bound (4.20), the continuity (4.5), the stability bound (4.18) and Theorem 3.2 (see (3.1)) we have

$$
\|\nabla \phi\|_{1 / 2+s, \Omega} \leq C\left(\left(1+C_{1}+C_{1} \omega\right)\|\boldsymbol{J}\|_{0, \Omega}+(1+\omega) C_{2}\|\boldsymbol{g}\|_{0, \partial \Omega}+\|\boldsymbol{g}\|_{s_{g}, \partial \Omega}\right)
$$

and consequently

$$
\|\nabla \times \boldsymbol{E}\|_{1 / 2+s, \Omega} \leq C\left(\left(1+C_{1}+C_{1} \omega\right)\|\boldsymbol{J}\|_{0, \Omega}+(1+\omega) C_{2}\|\boldsymbol{g}\|_{0, \partial \Omega}+\|\boldsymbol{g}\|_{s_{g}, \partial \Omega}\right) .
$$

The bounds (4.17) and (4.22) give the stability bound (4.11).

Remark 4.5. In the case of convex polyhedral domains, the smoothness parameters $s$ reaches the regularity of the boundary datum $s=s_{g}<1 / 2$, since Lemma 4.1 holds true for all $0<s_{\Omega}<1 / 2$, and $s^{*}=1$ in Lemma 4.2 (see footnote 2). 
Remark 4.6. The regularity of solutions stated in Theorem 4.4 guarantees that the tangential traces of $\boldsymbol{E}$ and $\nabla \times \boldsymbol{E}$ are in $L_{T}^{2}(\partial \Omega)$.

Remark 4.7. For $C^{2}$-domains, under all the other assumptions made in Theorem 4.4 the $H^{1}$-regularity of both $\boldsymbol{E}$ and $\nabla \times \boldsymbol{E}$ was already established in [8, Sect. 4.5.d] (see also Lemma 3.2 in this paper); the stability estimate

$$
\|\nabla \times \boldsymbol{E}\|_{1, \Omega}+\omega\|\boldsymbol{E}\|_{1, \Omega} \leq C\left((1+\omega)\left(\|\boldsymbol{J}\|_{0, \Omega}+\|\boldsymbol{g}\|_{0, \partial \Omega}\right)+\|\boldsymbol{g}\|_{1 / 2, \partial \Omega}\right)
$$

can be obtained along the lines of the proof of Theorem 4.4

\section{Conclusion}

In this paper, we have established some new regularity results and stability estimates for solutions to the time-harmonic Maxwell equations with impedance boundary conditions. More precisely, we have proved stability estimates with constant independent of the wavenumber for smooth domains and for polyhedral domains (see Theorem [3.1 and Theorem [3.2 respectively), and an elliptic regularity result for polyhedral domains (see Theorem 4.4).

Possible applications of these results are the convergence analysis of numerical methods for the approximation of Maxwell's equations and the stability analysis of electromagnetic boundary integral operators. For these applications, some extension of Theorem 3.1 (stability for smooth domains) might be interesting: $i$ ) to non-star shaped domains; in this case, in the definition of the test function $\boldsymbol{\xi}, \boldsymbol{x}$ should be substituted by a more general vector field; ii) to domains containing a (star-shaped) hole and with mixed boundary conditions, in order to extend to the Maxwell case the results proved in [11] for the Helmholtz problem; this would be the key point to extend to this case also all the other results proved for polyhedral domains; iii) to non-constant or anisotropic material coefficients $\epsilon$ and $\mu$; the key tool for this extension would be the use of more general Rellich identities, as the one introduced by Payne and Weinberger in [21] (see also [20, Sect. 5.1.1] and [16, Lemma 4.22]).

\section{Acknowledgment}

This section should come after the Appendices if any and should be unnumbered. Funding information may also be included here.

\section{References}

\section{References}

1. C. Amrouche, C. Bernardi, M. Dauge, and V. Girault, Vector potentials in three-dimensional non-smooth domains, Math. Methods Appl. Sci., 21 (1998), pp. 823864.

2. S. Axler, P. Bourdon, And W. Ramey, Harmonic function theory, Graduate Texts in Mathematics, Springer-Verlag, New York, 2001. 
3. A. Buffa And P. CiArlet, JR., On traces for functional spaces related to Maxwell's equations. I. An integration by parts formula in Lipschitz polyhedra, Math. Methods Appl. Sci., 24 (2001), pp. 9-30.

4. A. Buffa, M. Costabel, And C. Schwab, Boundary element methods for Maxwell's equations on non-smooth domains, Numer. Math., 92 (2002), pp. 679-710.

5. A. Buffa, M. Costabel, And D. Sheen, On traces for $\mathbf{H}(\mathbf{c u r l}, \Omega)$ in Lipschitz domains, J. Math. Anal. Appl., 276 (2002), pp. 845-867.

6. S. N. ChANDler-WILdE AND P. MONK, Wave-number-explicit bounds in timeharmonic scattering, SIAM J. Math. Anal., 39 (2008), pp. 1428-1455.

7. P. Cummings And X. Feng, Sharp regularity coefficient estimates for complex-valued acoustic and elastic Helmholtz equations, Math. Models Methods Appl. Sci., 16 (2006), pp. 139-160.

8. M. Dauge, M. Costabel, and S. NiCaise, Corner Singularities and Analytic Regularity for Linear Elliptic Systems. Part I: Smooth domains, Tech. Rep. 10-09, IRMAR, 2010. Available at http://perso.univrennes1.fr/monique.dauge/publis/CoDaNi_Analytic_Part_I.pdf.

9. V. Girault AND P.-A. Raviart, Finite element methods for Navier-Stokes equations, vol. 5 of Springer Series in Computational Mathematics, Springer-Verlag, Berlin, 1986. Theory and algorithms.

10. P. Grisvard, Elliptic problems in nonsmooth domains, vol. 24 of Monographs and Studies in Mathematics, Pitman (Advanced Publishing Program), Boston, MA, 1985.

11. U. Hetmaniuk, Stability estimates for a class of Helmholtz problems, Communications in Mathematical Sciences, 5 (2007), pp. 665-678.

12. R. Hiptmair, Finite elements in computational electromagnetism, Acta Numer., 11 (2002), pp. 237-339.

13. R. Hiptmair, A. Moiola, and I. Perugia, The Trefftz-DG method for the timeharmonic Maxwell equations. In preparation.

14. — Plane wave discontinuous Galerkin methods for the 2D Helmholtz equation: analysis of the p-version, Tech. Rep. 2009-20, SAM-ETH Zürich, 2009.

15. R. Hiptmair AND C. Schwab, Natural boundary element methods for the electric field integral equation on polyhedra, SIAM J. Numer. Anal., 40 (2002), pp. 66-86.

16. W. MCLEAn, Strongly elliptic systems and boundary integral equations, Cambridge University Press, Cambridge, 2000.

17. J. Melenk, On Generalized Finite Element Methods, PhD thesis, University of Maryland, USA, 1995.

18. P. Monk, Finite element methods for Maxwell's equations, Clarendon Press, Oxford, 2003.

19. P. Monk, Finite element methods for Maxwell's equations, Numerical Mathematics and Scientific Computation, Oxford University Press, New York, 2003.

20. J. NeČAs, Les méthodes directes en théorie des équations elliptiques, Masson et Cie, Éditeurs, Paris, 1967.

21. L. E. PAYNe AND H. F. WeInBERGER, New bounds for solutions of second order elliptic partial differential equations, Pacific J. Math., 8 (1958), pp. 551-573.

22. J.-P. Rosay And W. Rudin, A maximum principle for sums of subharmonic functions, and the convexity of level sets, Michigan Math. J., 36 (1989), pp. 95-111.

23. E. A. Spence, S. N. Chandler-Wilde, I. G. Graham, and V. P. Smyshlyaev, A new frequency-uniform coercive boundary integral equation for acoustic scattering. Report. Department of Mathematics, University of Bath. Submitted, 2010. 